\title{
Disformal gravity theories: A Jordan frame analysis
}

\author{
Jeremy Sakstein ${ }^{*}$ and Sarunas Verner ${ }^{\dagger}$ \\ Institute of Cosmology and Gravitation, University of Portsmouth, Portsmouth PO1 3FX, United Kingdom
}

(Received 25 September 2015; published 18 December 2015)

\begin{abstract}
The Jordan frame action for general disformal theories is presented and studied for the first time, motivated by several unresolved mysteries that arise when working in the Einstein frame. We present the Friedmann equations and, specializing to exponential functions, study the late-time cosmology using both dynamical systems methods and by finding approximate solutions. Our analysis reveals that either the disformal effects are irrelevant or the Universe evolves towards a phantom phase where the equation of state of dark energy is -3 . There is a marginal case where the asymptotic state of the Universe depends on the model parameters and de Sitter solutions can be obtained. Our findings indicate that the metric singularity found using the Einstein frame construction corresponds to phantom behavior in the Jordan frame and we argue that this is the case for general disformal theories.
\end{abstract}

DOI: 10.1103/PhysRevD.92.123005

PACS numbers: 95.36.+x, 04.50.Kd, 98.80.-k

\section{INTRODUCTION}

The elusive nature of dark energy has prompted theoretical interest in the cosmological dynamics of scalar fields (see [1] for a review) as a mechanism for driving the acceleration of the cosmic expansion. With the exception of the simplest models such as quintessence [2,3] and $k$ essence [4], theories that include an additional scalar are alternative theories of gravity [5] (see [6] for a recent compendium of cosmologically relevant theories); they include additional degrees of freedom that couple to matter, resulting in additional gravitational strength (or larger) interactions.

Many (but not all) modified gravity models can be written in the schematic form

$S=\int \mathrm{d}^{4} x \sqrt{-g}\left[\mathcal{L}_{g}\left(g_{\mu \nu}\right)+\mathcal{L}_{\phi}(\phi)\right]+S_{\mathrm{m}}\left[\tilde{g}_{\mu \nu}\left(g_{\mu \nu}, \phi\right)\right]$,

where the scalar field $\phi$ is taken to be dimensionless. This action describes a theory of gravity in the so-called Einstein frame. $\mathcal{L}_{g}$ contains tensor self-interactions of $g_{\mu \nu}$ through curvature tensors such as the Ricci scalar and $\mathcal{L}_{\phi}$ contains scalar self-interactions. No direct couplings of the scalar to curvature tensors are present and the modifications of general relativity (GR) are encoded in the coupling to matter. In particular, test bodies do not move on geodesics of $\tilde{g}_{\mu \nu}$, the Einstein frame metric, but instead respond to the composite metric $\tilde{g}_{\mu \nu}\left(g_{\mu \nu}, \phi\right)$ - the Jordan frame metric. It was shown by Bekenstein $[7,8]$ that the most general theory of a scalar coupled to matter that preserves causality is

\footnotetext{
*jeremy.sakstein@ port.ac.uk sn3g11@soton.ac.uk
}

$$
\begin{aligned}
\tilde{g}_{\mu \nu} & =C(\phi, X) g_{\mu \nu}+D(\phi, X) \partial_{\mu} \phi \partial_{\nu} \phi ; \\
X & \equiv-\frac{1}{2} g^{\mu \nu} \partial_{\mu} \phi \partial_{\nu} \phi .
\end{aligned}
$$

Indeed, it has been shown that theories where matter is coupled to metrics of this form are free of the Ostrogradski ghost instability [9-19]. $C(\phi, X)$ is known as the conformal factor, and its consequences have been well studied, at least when it depends on $\phi$ only. Consequently, $D(\phi, X)$ has become known as the disformal factor and the term $D \phi_{\mu} \phi_{\nu}$, the disformal coupling to matter, or simply the disformal part of the metric. Any theory where $D(\phi, X) \neq 0$ falls into the class of disformal gravity theories.

Disformal couplings are ubiquitous in fundamental physics. They arise in the low energy effective action of string theory [20] and are linked to Galileons through probe branes moving in higher dimensional space-times [21,22]. They also arise in the decoupling limit of massive gravity [23]. In the context of Horndeski theories [13,24], the most general scalar-tensor theories with second-order equations of motion, they are the most general transformation that preserve the form of the scalar-tensor sector when $C$ and $D$ depend on $\phi$ only [14]. These are the motivation behind a recent phenomenological study of disformal theories in several different contexts [20,25-42], with most attention focusing on the case where $C$ and $D$ depend on $\phi$ only.

The parametrized post-Newtonian (PPN) parameters for this class of disformal theories were calculated in [33,42], where it was shown that they are completely determined by the cosmological scalar $\phi_{0}$. For this reason, knowledge of the cosmology of these theories is vital for determining their viability. The first steps towards this were made by the authors of [34], who used dynamical system techniques to classify the cosmological solution space in the Einstein frame with the goal of identifying models that passed solar 
system bounds. Several new fixed points were found but all had one problem in common: the Jordan frame metric becomes increasingly singular as the fixed point is approached, corresponding to the lapse in the Jordan frame approaching zero. This may represent several pathologies with the theory including the lack of a nonrelativistic limit and a freezing of the proper time for observers, all of which we will discuss in more detail in the next section.

Currently, it is unclear whether the singularity is debilitating for the theory or if it is merely an artifact of working in the Einstein frame. The two frames are equivalent for calculational purposes provided that the solutions are interpreted appropriately when relating them to observations. Furthermore, one cannot interpret observations until the proper time for an observer is aligned with the coordinate time and, since matter moves on geodesics of the Jordan frame metric, this frame is singled out for observations ${ }^{1}$ [42]. Finally, Wetterich [43] has shown that frame transformations may introduce spurious solutions that solve the field equations in one frame but not the other. Given this, a study of the Jordan frame cosmology with a view to addressing these outstanding issues is certainly merited.

This is the purpose of this paper. In the next section, we introduce disformal gravity theories in the Einstein frame and discuss the nature of the singularity, including the associated pathologies, in more detail. The applicability of the Einstein frame dynamical system to fundamental observers is also discussed. We next move on to study the Jordan frame cosmology. In Sec. III we present the Jordan frame Friedmann and Klein-Gordon equations and use them to develop a dynamical systems approach to classifying the solutions for exponential scalar potentials and disformal factors. We find that theories where the disformal factor is small (in a manner to be made precise below) behave in a similar manner to quintessence but theories where the disformal factor is large are not well described by a dynamical systems analysis in the sense that the fixed points reveal little about the late-time cosmology. Instead, we focus on finding exact solutions at late times in Sec. V. Here, we show that the theory exhibits phantom behavior at late times with an effective dark energy equation of state $w=-3$.

Models that exhibit phantom behavior are precisely those that suffer from singularities in the Einstein frame and thus we conclude that the singularity is indeed a physical pathology, the Jordan frame manifestation being phantom behavior. There is a marginal case that corresponds to a specific tuning in the parameter space of the theory. In this case, the asymptotic state of the Universe is a function of the model parameters and we show that it is possible to achieve asymptotically de Sitter solutions using

\footnotetext{
${ }^{1}$ Note that we do not claim that one frame is any more physical than the other, only that the Jordan frame is the frame where the coordinate time is aligned with the proper time for an observer, thus making any calculations simple to compare with other alternate gravity theories.
}

a suitable tuning. We discuss our findings and conclude in Sec. VI. In particular, we argue that the qualitative features we observer here-quintessence fixed points and phantom behavior-are features of general disformal models.

For the reader interested purely in the cosmology of disformal models and not the singular nature of the disformal transformation, the Jordan frame cosmology is presented here for the first time and can be found in Sec. III onwards. The Friedmann equations here can be used directly for computing quantities such as the luminosity distance-redshift relation, which requires a transformation to coordinates appropriate for comoving observers if one uses the Einstein frame. Furthermore, the nonphantom regions of the parameter space of exponential models are presented in the conclusions (Sec. VI) where we also discuss the application to more general models.

\section{THE EINSTEIN FRAME}

In this section we present the Einstein frame action we will consider and use it to describe the singularity in the Jordan frame as well as the potential pathologies it presents. The action we will consider is

$$
\begin{aligned}
S= & \int \mathrm{d}^{4} x \sqrt{-g} M_{\mathrm{pl}}^{2}\left[\frac{R(g)}{2}-\frac{1}{2} \nabla_{\mu} \phi \nabla^{\mu} \phi-V(\phi)\right] \\
& +S_{\mathrm{m}}\left[\tilde{g}_{\mu \nu}\right] .
\end{aligned}
$$

The Jordan frame metric is

$$
\tilde{g}_{\mu \nu}=g_{\mu \nu}+\frac{B^{2}(\phi)}{\Lambda^{2}} \partial_{\mu} \phi \partial_{\nu} \phi
$$

Specializing to the case of a flat Friedmann-RobertsonWalker (FRW) space-time,

$$
\mathrm{d} s_{\mathrm{E}}^{2}=-\mathrm{d} t_{\mathrm{E}}^{2}+a_{\mathrm{E}}^{2} \mathrm{~d} x_{\mathrm{E}}^{2},
$$

where we use the subscripts $\mathrm{E}$ and $\mathrm{J}$ to represent Einstein and Jordan frame quantities respectively, the Jordan frame singularity can be seen by computing the metric determinants [44]:

$$
\frac{\sqrt{-\tilde{g}}}{\sqrt{-g}}=\sqrt{1-\Sigma} ; \quad \Sigma \equiv \frac{B^{2}(\phi)}{\Lambda^{2}}\left(\frac{\mathrm{d} \phi}{\mathrm{d} t_{\mathrm{E}}}\right)^{2} .
$$

When $\Sigma=1$ the Jordan frame metric is singular. Using (4), the lapse in the Jordan frame is

$$
N^{2}=1-\Sigma
$$

so that

$$
\mathrm{d} t_{\mathrm{J}}=N \mathrm{~d} t_{\mathrm{E}}
$$


One can see that, cosmologically, the metric singularity corresponds to this becoming zero.

There are several physical issues with the approach to the singularity. First, the Jordan frame space-time is

$$
\mathrm{d} \tilde{s}^{2}=-N^{2} \mathrm{~d} t_{\mathrm{E}}^{2}+a(t)^{2} \mathrm{~d} x^{2}
$$

and so the proper time $\tau$ for physical observers is [42]

$$
\frac{\mathrm{d} \tau}{\mathrm{d} t_{\mathrm{J}}}=N,
$$

and so an observer's proper time is frozen at the singularity. Furthermore, since $\tilde{g}_{\mu \nu} u^{\mu} u^{\nu}=-1$, the Lorentz factor is [42]

$$
\gamma=\frac{1}{N}\left(1-\frac{v^{2}}{c^{2}}\right)
$$

where $v^{i}=\mathrm{d} x^{i} / \mathrm{d} t_{\mathrm{J}}$. Typically, one derives the Newtonian behavior of the theory by looking at the limit $v / c \ll 1$. In this case however, this is not sufficient. As demonstrated in [42], one also requires $\Sigma \ll 1$ in order to have a sensible postNewtonian expansion. Since the singularity corresponds precisely to $\Sigma \rightarrow 1$, this behavior is lost as the singularity is approached and there is no sensible Newtonian limit. The lack of such a limit was also noted in $[33,34]$ using Einstein frame coordinates. In this case, Newtonian quantities such as the total force diverge as $\Sigma \rightarrow 1$. Since $N$ is also the ratio of the speed of light to that of tensors, the Einstein frame interpretation of this is that there are no particles that move with nonrelativistic velocities in this limit.

One obvious question is then, why not use FRW coordinates with unit lapse from the outset in the Jordan fame? In this case there is no apparent singularity at the level of the metric and any potential pathology must appear through the solution of the Friedmann equations. Indeed, our choice of coordinates such that $g_{\mu \nu}$ is FRW is not a choice of space-time since no particles follow geodesics of $g_{\mu \nu}$. Applying the change of time coordinate (8) to (9) one has

$$
\mathrm{d} \tilde{s}^{2}=-\mathrm{d} t_{\mathrm{J}}^{2}+a\left(t_{\mathrm{J}}\right)^{2} \mathrm{~d} x^{2}
$$

where $a\left(t_{\mathrm{J}}\right)=a\left(t_{\mathrm{J}}\left(t_{\mathrm{E}}\right)\right)$. This is a FRW space-time and so one can see that the singularity found taking $g_{\mu \nu}$ to be FRW is simply a coordinate singularity. ${ }^{2}$ The one remaining question

\footnotetext{
${ }^{2}$ The reader should note that this is strictly true in the context of an isotropic and homogeneous cosmology. Whether or not there are other physical scenarios where a nonremovable Jordan frame singularity is present is unknown, although we note that, to date, none has been observed. All of the pathologies that arise due to the singularity discussed in this section are the result of the cosmological singularity and the aim of this work is to understand the physical implications of this. For this reason, we focus entirely on the cosmological singularity and will not attempt to address the more general question of potential singularities elsewhere.
}

is that of $a\left(t_{\mathrm{J}}\right)$. Currently, it is not known whether or not the transformation (8) introduces any singularities into the spatial part of the metric. Said another way, is there some finite time $\bar{t}_{\mathrm{J}}$ such that $a\left(\bar{t}_{\mathrm{J}}\right)=0$ ? This is a difficult question if one begins in the Einstein frame. Equation (8) is highly nonlinear, and one requires an exact solution to provide an answer. Conversely, the Jordan frame is a perfect tool because one can classify the entire cosmological solution space using dynamical systems or other techniques. One then has the cosmological information that can be compared to data, as well as knowledge of any pathologies. One can identify the Jordan frame coordinates corresponding to the singularity found using the coordinates (5) because applying the transformation (8) one finds

$$
N^{2}=\left[1+\frac{B^{2}}{\Lambda^{2}}\left(\frac{\mathrm{d} \phi}{\mathrm{d} t_{\mathrm{J}}}\right)^{2}\right]^{-1}
$$

and so in these coordinates the singularity corresponds to $B \mathrm{~d} \phi / \mathrm{d} t_{\mathrm{J}} / \Lambda \rightarrow \infty$. Another advantage of working in the Jordan frame exclusively is the following. Wetterich [43] has pointed out that spurious solutions can exist whereby a specific solution may be a solution of the Einstein frame equations of motion but not the Jordan frame equations. This potential problem is mitigated by working in the Jordan frame from the outset.

All of the potential problems discussed above clearly motivate our study of the Jordan frame cosmology of disformal theories. Ultimately, we will see that when $\mathrm{d} \phi / \mathrm{d} t_{\mathrm{J}} \rightarrow \infty$ i.e. in the limit where the singularity is present in Einstein frame time, the Universe undergoes strong phantom behavior ( $w=-3$ for exponential models) and therefore the pathologies associated with the singularity are physical, the Jordan frame manifestation being precisely said phantom behavior. In terms of the Einstein frame coordinates (5) one can see that as the singularity is approached, the Jordan frame lapse tends increasingly towards zero and therefore, for comoving observers, a large number of $e$-folds can pass in a small amount of proper time. When viewed in this manner, the phantom behavior is hardly surprising.

Before moving on to study the Jordan frame, we end this section by discussing the use of dynamical systems in both frames. The Einstein frame dynamical system for exponential models was studied in $[27,34]$. In order to achieve an autonomous system of equations, one uses the variable $N_{\mathrm{E}}=\ln a_{\mathrm{E}}$ as a time variable and chooses appropriate variables $x_{i}$ that span the phase space of the system. Fixed points correspond to points in the phase space where

$$
\frac{\mathrm{d} x_{i}}{\mathrm{~d} N_{\mathrm{E}}}=0 \quad \forall i
$$

It is assumed that $t_{\mathrm{E}}$ is a monotonic function of $N_{\mathrm{E}}$ and that $N_{\mathrm{E}} \rightarrow \infty \Rightarrow t_{\mathrm{E}} \rightarrow \infty$, both of which are necessary for 
global attractors of the dynamical system to correspond to the asymptotic state of the system (see $[45,46]$ for the more technical aspects of dynamical systems theory). Note however that $t_{\mathrm{E}} \rightarrow \infty$ does not necessarily imply that $t_{\mathrm{J}} \rightarrow \infty$. This depends on being able to integrate (8) exactly and so fixed points in the Einstein frame do not necessarily correspond to the asymptotic future in the Jordan frame. One case where this can be achieved trivially is the case where $N=1$ at the fixed point. In this case, disformal effects are absent and the theory behaves in an identical manner to quintessence. Away from this limit, there are some important and physically relevant quantities that cannot be calculated using the Einstein frame. One pertinent example of this is the Hubble constant

$$
H_{\mathrm{J}}=\frac{H_{\mathrm{E}}}{N} .
$$

In the Einstein frame, $H_{\mathrm{E}} \rightarrow 0$ at the fixed points and there are also fixed points where $N \rightarrow 0$ in the same limit. These are fixed points corresponding to the singularity. This means that the behavior of $H_{\mathrm{J}}$ is undetermined. If the phase space were one dimensional, one could simply use l'Hôpital's rule to find the asymptotic value but there is no higher dimensional analogue of this theorem. For this reason, the asymptotic value depends on the phase space trajectory of the specific solution as it approaches the fixed point. In this case, the Einstein frame dynamical system fails to achieve its goal of predicting the universal late-time behavior since knowledge of the fixed points alone is not sufficient to know the asymptotic state of the Universe. This is an artifact of working in a coordinate system where the asymptotic state of the dynamical system does not correspond to the limit of infinite proper time as seen by comoving observers. ${ }^{3}$ Conversely, the Jordan frame dynamical system is perfectly able to predict all of the physically relevant quantities precisely because the coordinates are FRW with unit lapse from the outset.

\section{THE JORDAN FRAME}

From here on we work exclusively in the Jordan frame. For this reason, we will drop all unnecessary subscripts and tildes; it is to be understood that all quantities are Jordan frame quantities. The Jordan frame action is complicated compared with the simplicity of its Einstein frame counterpart, as is the derivation of the field equations. For this reason, we give the calculation of the Jordan frame action and the field equations in Appendix A and present the final results here.

\footnotetext{
${ }^{3}$ One may wonder whether it is possible to choose Einstein frame coordinates to avoid this problem. Such a choice of coordinates would require working in a coordinate system where $\phi(t)$ is part of the Einstein frame metric and would ultimately require one to mix frame variables in the equations of motion.
}

We begin by defining the disformal coupling,

$$
\beta=\frac{\mathrm{d} \ln B(\phi)}{d \phi} .
$$

In general, $\beta$ can be an arbitrary function of $\phi$ but, in what follows, we set $\beta$ to be constant so that

$$
B(\phi)=e^{\beta \phi} .
$$

Second, the scalar potential is

$$
V(\phi)=m_{0}^{2} e^{-\lambda \phi},
$$

where $\lambda$ is a constant and $m_{0}$ is an a priori arbitrary, mass scale. These choices are made so that the equations exhibit a scaling symmetry that allows for the existence of scaling solutions and hence the dimension of the phase space is minimal [34]. With these newly defined constants, one can start building the disformal model. First, we need the Friedmann equations:

$$
\begin{gathered}
3 H^{2}=\frac{\dot{\phi}^{2}}{2}+V u+8 \pi G \rho_{m} u^{3 / 2} \\
\dot{H}=-\frac{\dot{\phi}^{2}}{2}-4 \pi G \rho_{m} u^{3 / 2}+\frac{B^{2} H}{u \Lambda^{2}}\left(\beta \dot{\phi}^{3}+\dot{\phi} \ddot{\phi}\right),
\end{gathered}
$$

which are derived in Appendix A 2. The variable $u$ is defined for notational convenience and is given by

$$
u=1+\frac{B^{2} \dot{\phi}^{2}}{\Lambda^{2}} .
$$

One can already see the advantage of working in the Jordan frame; the Friedmann constraint contains the disformal scale $\Lambda$ and so it is possible to compactify the phase space without using unphysical variables. This is in stark contrast to the Einstein frame, where the Friedmann constraint is identical to that of the equivalent quintessence theory and it is necessary to use advanced techniques relating to fixed points at infinity to determine the late-time dynamics [34]. Next, we need the scalar equation of motion, which can be expressed as

$$
\begin{aligned}
\ddot{\phi} & +\left[\frac{8 \pi G \rho_{m} B^{2}}{\Lambda^{2}}\left(\ddot{\phi}+\beta \dot{\phi}^{2}\right)\right] u^{3 / 2}+V_{\phi} u^{2}+3 H \dot{\phi} u \\
& =\frac{\beta B^{2} \dot{\phi}^{4}}{\Lambda^{2}} .
\end{aligned}
$$

Because we are working in the Jordan frame, the scalar is minimally coupled to matter and one has the usual continuity equation:

$$
\dot{\rho}_{\mathrm{m}}+3 H \rho_{\mathrm{m}}=0 .
$$


Equations (19)-(23) contain all the necessary information about the dynamics of the system. The complexity of these equations makes it impossible to find exact analytic solutions and one method to analyze their late-time behavior is to use a dynamical systems analysis. These methods are not new in cosmology. Indeed, they were applied previously to study quintessence [47] and disformal theories in the Einstein frame [34]. Moreover, a dynamical systems analysis is a powerful tool to calculate the late-time cosmological observables. The unfamiliar reader can find an introduction to dynamical systems and their use in cosmology in $[1,34,45,46,48-50]$.

Before proceeding to formulate the equations as a dynamical system, we pause to discuss the physical observables we wish to calculate using our subsequent analysis. Typically one is interested in the dark energy density parameter $\Omega_{\mathrm{DE}}$ and the equation of state $w$. These are difficult to define and several inequivalent effective variables are often found in the literature. ${ }^{4}$ Instead of defining effective quantities, we will look for quantities whose definition and interpretation are insensitive to the theory of gravity. ${ }^{5}$ One suitable quantity is the deceleration parameter

$$
q=-\frac{\ddot{a} a}{\dot{a}^{2}},
$$

which implies that

$$
\frac{\dot{H}}{H^{2}}=-(1+q)
$$

independent of the theory of gravity. In the case of $w \mathrm{CDM}$, one has

$$
q=\frac{1}{2}\left(1+3 w_{\mathrm{eff}}\right)
$$

where $w_{\text {eff }}{ }^{6}$ depends on both $w_{\mathrm{m}}$ and $w_{\mathrm{DE}}$. This motivates the definition

$$
w_{\text {eff }}=-1-\frac{2}{3} \frac{\dot{H}}{H^{2}} .
$$

$q<0$, or equivalently, $w_{\text {eff }}<1 / 3$ indicates that the cosmic expansion is accelerating and so we will use these to classify the nature of the solutions. Formally, one may define

$$
\Omega_{\mathrm{DE}} \equiv 1-\Omega_{\mathrm{m}}
$$

\footnotetext{
${ }^{4}$ See $[37,51-55]$ for discussions relating to effective quantities in scalar-tensor theories.

${ }^{5}$ By which we mean they describe properties of the FRW metric and are not found by comparing the Friedmann equations to those resulting from the Einstein-Hilbert action.

${ }^{6}$ We use the notation eff to denote a single composite quantity that describes the evolution of the Universe and not an effective equation of state for dark energy.
}

and we will often refer to this quantity but the reader should be aware that this is not the same quantity that is inferred from cosmic microwave background or luminosity distance measurements ${ }^{7}$; it is merely an indication of what is driving the evolution of the Universe.

\section{FORMULATION AS A DYNAMICAL SYSTEM}

In this section, we formulate the Friedmann-KleinGordon equations as a dynamical system and classify the fixed points.

\section{A. Construction of the phase space}

In order to make contact with the quintessence literature, we begin by introducing the new variables

$$
x \equiv \frac{\phi^{\prime}}{\sqrt{6}}, \quad \text { and } \quad y \equiv \frac{\sqrt{V}}{\sqrt{3} H},
$$

where instead of differentiating with respect to coordinate time $t$, we differentiate with respect to $N \equiv \ln a(t)$. We denote derivatives with respect to $N$ using a prime. This coordinate choice allows us to reduce the dimension of the phase space by 1 . Using these variables, we can rewrite the Friedmann constraint as

$$
1=x^{2}+y^{2} u+\Omega_{\mathrm{m}} u^{3 / 2} .
$$

As noted in [34], the disformal phase space is threedimensional and so we require one more variable to close the system. The authors of [34] chose the variable $z=$ $B H / \Lambda$ but this results in a phase space that is noncompact. In particular, note that $u=1+6 x^{2} z^{2}$ in this system so that neither the $x$ - nor the $z$-directions are compact owing to the fact that $\Omega_{\mathrm{m}}$ can be arbitrarily small. Instead, one can work in a compact phase space by introducing the following variables:

$$
X \equiv x u^{-\frac{3}{4}}, \quad Y \equiv y u^{-\frac{1}{4}} \quad Z \equiv u^{-\frac{3}{4}} .
$$

When written in terms of these new variables, the Friedmann constraint becomes

$$
Z^{2}=X^{2}+Y^{2}+\Omega_{m} .
$$

This implies that $-1 \leq X \leq 1,0 \leq Y \leq 1$ and $0 \leq Z \leq 1$; therefore the phase space is compact. When written in terms of these variables, the phase space is a half-cone with the vertex located at $(0,0,0)$. This is shown in Fig. 1. Its base is the semicircle $x^{2}+y^{2}+\Omega_{\mathrm{m}}=1$ located in the plane $Z=1$. This is precisely the phase space of quintessence

\footnotetext{
${ }^{7} \mathrm{By}$ this, we mean that the values of $w$ and $\Omega_{\mathrm{DE}}$ are found by fitting the data to functional forms where $w$ is constant, which is not necessarily the case for disformal models.
} 


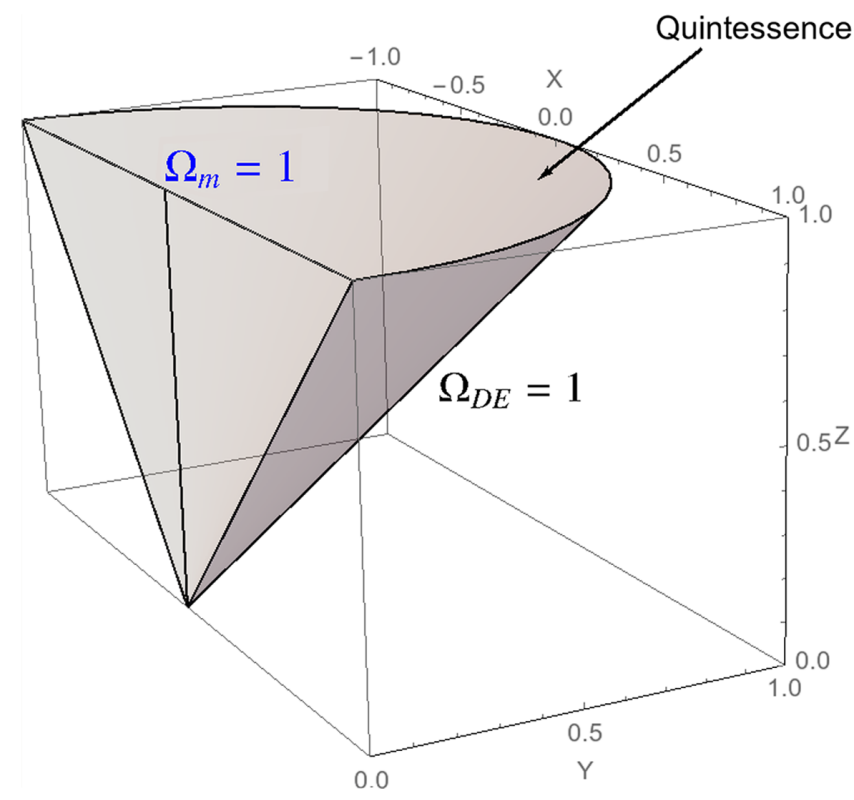

FIG. 1 (color online). The phase space of the system. The point $(0,0,1)$ corresponds to matter dominated solutions and the edges of the cone correspond to dark energy dominated solutions. The phase space of quintessence coincides with the base of the cone located in the $Z=1$ plane.

and hence corresponds to the subset of the theory where disformal effects are absent. Any fixed points that lie on the base of the cone therefore have late-time cosmologies that are identical to those found for pure quintessence theories with an exponential potential [48]. Note, however, that their stability may be altered since the three-dimensional phase space implies the existence of a third eigenvalue, and that the other two eigenvalues may assume different values from those found in a purely two-dimensional phase space. Setting $\Omega_{\mathrm{m}}=0$, one can see that the sides of the cone correspond to dark energy dominated solutions whereas setting $X=Y=0$, one has $\Omega_{\mathrm{m}}=Z=1$, and so the point $(0,0,1)$ corresponds to a matter dominated solution. We therefore expect all physical trajectories to originate from its vicinity.
Special attention must be paid to the tip of the cone $X=Y=Z=0$, which corresponds to what would be the metric singularity had we worked in the Einstein frame. This is a peculiar point because a fixed point here tells us absolutely nothing about the late-time cosmology. Typically, fixed points such as these indicate that the effective dimension of the phase space is reduced and one typically requires center manifold methods to find the reduced phase space. Indeed, these methods are necessary for analyzing the cosmology in the Einstein frame [34]. The reduced phase space is often unphysical, ${ }^{8}$ and an alternate approach is to look for approximate late-time solutions given that one has some idea of which terms in the equations can be ignored at late times. This is the approach that we will adopt in Sec. V.

The case $\beta=\lambda / 2$ was identified in [34] as a special parameter tuning in the Einstein frame where the dimension of the phase space is reduced to 2. This remains the case in the Jordan frame, where one has

$$
V(\phi) B^{2}(\phi)=m_{0}^{2},
$$

which implies a relation between $X, Y$, and $Z$ :

$$
Y^{2}=2 \frac{m_{0}^{2}}{\Lambda^{2}} \frac{X^{2}}{1-Z^{\frac{4}{3}}} .
$$

This relation is an additional constraint that must be satisfied and hence only two of the variables are independent. In terms of the three-dimensional phase space, the dynamics of the system are restricted to the twodimensional surface where (34) is satisfied and hence the phase space is two dimensional. For this reason the dynamics of this case must be treated separately.

Using Eq. (32) to eliminate $\Omega_{\mathrm{m}}$, Eqs. (19)-(22) can be expressed as a system of three autonomous first-order differential equations:

$$
\begin{aligned}
\frac{d X}{d N}= & \frac{X\left[X^{4}\left(3-9 Z^{\frac{4}{3}}\right)+6 X^{2} Z^{\frac{4}{3}}\left(Y^{2}-3 Z^{\frac{2}{3}}+4 Z^{2}\right)+3\left(Z^{4}-1\right)\left(Y^{2}-Z^{2}\right)^{2}\right.}{2 Z^{2}\left(X^{2}\left(3 Z^{4}-1\right)+\left(Z^{4}-1\right)\left(Z^{2}-Y^{2}\right)\right)} \\
& +\frac{\left.\sqrt{6} X Z\left(\lambda Y^{2}\left(3-5 Z^{4}\right)+2 \beta\left(Z^{4}-1\right)(Y-Z)(Y+Z)\right)-4 \sqrt{6} \beta X^{3} Z\left(Z^{4}-1\right)\right]}{2 Z^{2}\left(X^{2}\left(3 Z^{4}-1\right)+\left(Z^{4}-1\right)\left(Z^{2}-Y^{2}\right)\right)} \\
\frac{d Y}{d N}= & \frac{Y\left[X^{4}\left(3-9 Z^{\frac{4}{3}}\right)+6 X^{2} Z^{\frac{4}{3}}\left(Y^{2}-3 Z^{\frac{2}{3}}+2 Z^{2}\right)+3\left(Z^{4}-1\right)\left(Y^{2}-Z^{2}\right)^{2}\right)}{2 Z^{2}\left(X^{2}\left(3 Z^{4}-1\right)+\left(Z^{4}-1\right)\left(Z^{2}-Y^{2}\right)\right)} \\
& -\frac{\left.\sqrt{6} X^{3} Z\left(\lambda+6 \beta\left(Z^{4}-1\right)-3 \lambda Z^{\frac{4}{3}}\right)-\sqrt{6} \lambda X\left(Z^{\frac{4}{3}}-1\right) Z\left(2 Y^{2}+Z^{2}\right)\right]}{2 Z^{2}\left(X^{2}\left(3 Z^{\frac{4}{3}}-1\right)+\left(Z^{4}-1\right)\left(Z^{2}-Y^{2}\right)\right)}
\end{aligned}
$$

\footnotetext{
${ }^{8}$ In the sense that the variables are far removed from the underlying dynamical quantities such as $H$ and $\dot{\phi}$. The phase space still contains all of the late-time trajectories.
} 


$$
\frac{d Z}{d N}=-\frac{3 X\left(Z^{4}-1\right)\left(\sqrt{6}\left(2 \beta X^{2}+\lambda Y^{2}\right)-6 X Z\right)}{2 Z^{4}\left(X^{2}\left(1-3 Z^{\frac{4}{3}}\right)+\left(Z^{\frac{4}{3}}-1\right)\left(Z^{2}-Y^{2}\right)\right)} .
$$

Using Eq. (20), one finds

$$
\frac{H^{\prime}}{H}=\frac{X^{4}\left(9 Z^{\frac{4}{3}}-3\right)+4 \sqrt{6} \beta X^{3}\left(Z^{4}-1\right) Z-6 X^{2} Z^{\frac{4}{3}}\left(Y^{2}-2 Z^{\frac{2}{3}}+Z^{2}\right)+2 \sqrt{6} \lambda X Y^{2}\left(Z^{\frac{4}{3}}-1\right) Z-3\left(Z^{4}-1\right)\left(Y^{2}-Z^{2}\right)^{2}}{2 Z^{2}\left(X^{2}\left(1-3 Z^{\frac{4}{3}}\right)+\left(Z^{4}-1\right)\left(Z^{2}-Y^{2}\right)\right)},
$$

which can be used to calculate $w_{\text {eff }}$ and $q$. One also has $\Omega_{\mathrm{DE}}=1-Z^{2}+X^{2}+Y^{2}$ in these variables.

\section{B. Fixed points when $\beta \neq \lambda / 2$}

There are a total of five fixed points of Eqs. (35)-(37) that we list in Table I. Table II lists the interesting cosmological quantities at each point. The corresponding eigenvalues are listed below; only points (4) and (5) can be late-time attractors:

(1) $e_{1}=\frac{3}{2}, e_{2}=\frac{3}{2}, e_{3}=0$

(2) $e_{1}=3, e_{2}=-2 \sqrt{6} \beta-6, e_{3}=3+\sqrt{\frac{3}{2}} \lambda$

(3) $e_{1}=3, e_{2}=2 \sqrt{6} \beta-6, e_{3}=3-\sqrt{\frac{3}{2}} \lambda$

(4) $e_{1}=\lambda(2 \beta-\lambda), e_{2}=\frac{1}{2}\left(\lambda^{2}-6\right), e_{3}=\lambda^{2}-3$

(5) $e_{1}=\frac{6 \beta}{\lambda}-3, e_{2}=-\frac{3}{4}\left(1+\frac{\sqrt{24-7 \lambda^{2}}}{\lambda}\right), e_{3}=-\frac{3}{4}\left(1-\frac{\sqrt{24-7 \lambda^{2}}}{\lambda}\right)$.

Interestingly, not all solutions are fixed points. Point (1) is actually a fixed line, hence the zero eigenvalue. As discussed above, we will deal with this point using late-time solutions rather than dynamical systems. We note that $X=Y=Z=0$ is an independent fixed point not shown in the table. It corresponds to a matter

TABLE I. The fixed points of the system (35)-(37) when $\beta \neq \lambda / 2$.

\begin{tabular}{lcccc}
\hline \hline Name & $\mathrm{X}$ & $\mathrm{Y}$ & $\mathrm{Z}$ & Existence \\
\hline$(1)$ & 0 & 0 & $0<Z \leq 1$ & All \\
$(2)$ & -1 & 0 & 1 & All \\
$(3)$ & 1 & 0 & 1 & All \\
$(4)$ & $\frac{\lambda}{\sqrt{6}}$ & $\sqrt{1-\frac{\lambda^{2}}{6}}$ & 1 & $\lambda<\sqrt{6}$ \\
$(5)$ & $\frac{\sqrt{\frac{3}{2}}}{\lambda}$ & $\frac{\sqrt{\frac{3}{2}}}{\lambda}$ & 1 & Any \\
\hline \hline
\end{tabular}

TABLE II. The cosmological variables at the fixed points of the system (35)-(37) when $\beta \neq \lambda / 2$.

\begin{tabular}{lcccc}
\hline \hline Name & $H^{\prime} / H$ & $q$ & $w_{\text {eff }}$ & $\Omega_{\mathrm{DE}}$ \\
\hline$(1)$ & $-\frac{3}{2}$ & $\frac{1}{2}$ & 0 & 1 \\
$(2)$ & -3 & 2 & 1 & 1 \\
$(3)$ & -3 & 2 & 1 & 1 \\
$(4)$ & $-\frac{\lambda^{2}}{2}$ & $\frac{1}{2}\left(\lambda^{2}-2\right)$ & $\frac{1}{3}\left(\lambda^{2}-3\right)$ & 1 \\
$(5)$ & $-\frac{3}{2}$ & $\frac{1}{2}$ & 0 & $3 / \lambda^{2}$ \\
\hline \hline
\end{tabular}

dominated solution ${ }^{9}$ and so cosmologically viable trajectories should begin near this point. One can see that it is a saddle point and so trajectories will eventually leave its vicinity, signaling the onset of dark energy domination.

Points (2) and (3) are unstable nodes or saddle points that correspond to nonaccelerating phases and so we will pay no further attention to them. Points (4) and (5) are both located in the plane $Z=1$, which, as discussed above, corresponds to a quintessence subset. These fixed points are hence identical to the points found if one considers quintessence with an exponential potential in GR. In particular, point (4) is the dark energy dominated point that exists when $\lambda<\sqrt{6}$. Point (5) exists when $\lambda>\sqrt{6}$ and exhibits a matterlike behavior with $w_{\text {eff }}=0$. Unlike the case of GR, these points are not always stable when they exist. Indeed, one can see that both are unstable when $2 \beta>\lambda$. When this is the case, the only stable fixed point is at the tip of the cone and the dynamical systems analysis does not reveal anything interesting about the late-time dynamics. Examples of this are shown below in Figs. 2 and 3. If the theory was GR and quintessence, the models with $\lambda=1$ and $\lambda=4$ should approach fixed points (4) and (5) independently of the other parameters. These models are plotted in Fig. 2 with $m_{0}=$ $\Lambda=H_{0}$ and $\beta<\lambda / 2$ (the model parameters are indicated in the captions). One can see that these points are eventually reached after a brief excursion into the domain $Z<1$. In Fig. 3 we plot the same models but instead choose $\beta>\lambda / 2$. Once can see that, in this case, both models now evolve towards the tip of the cone.

One can then conclude that models with $\beta<\lambda / 2$ have late-time cosmologies that are identical to quintessence whereas those with $\beta>\lambda / 2$ exhibit drastically different behavior. We will calculate this below in Sec. V but we note here for completeness that points (2)-(5) are identical to those found in [34]. ${ }^{10}$ The reason for this is that, as discussed in Sec. II, these points all have $Z=1$, which corresponds to $B \dot{\phi} / \Lambda=0$ i.e. no disformal coupling. In this limit, the

\footnotetext{
${ }^{9}$ This is point (1) Table $\mathrm{I}$ in [34] and the first bullet point Appendix C in [27], when the conformal parameter $\alpha=0$ (in both cases).

${ }^{10}$ These are points (2)-(5) in that reference, Table I.
} 


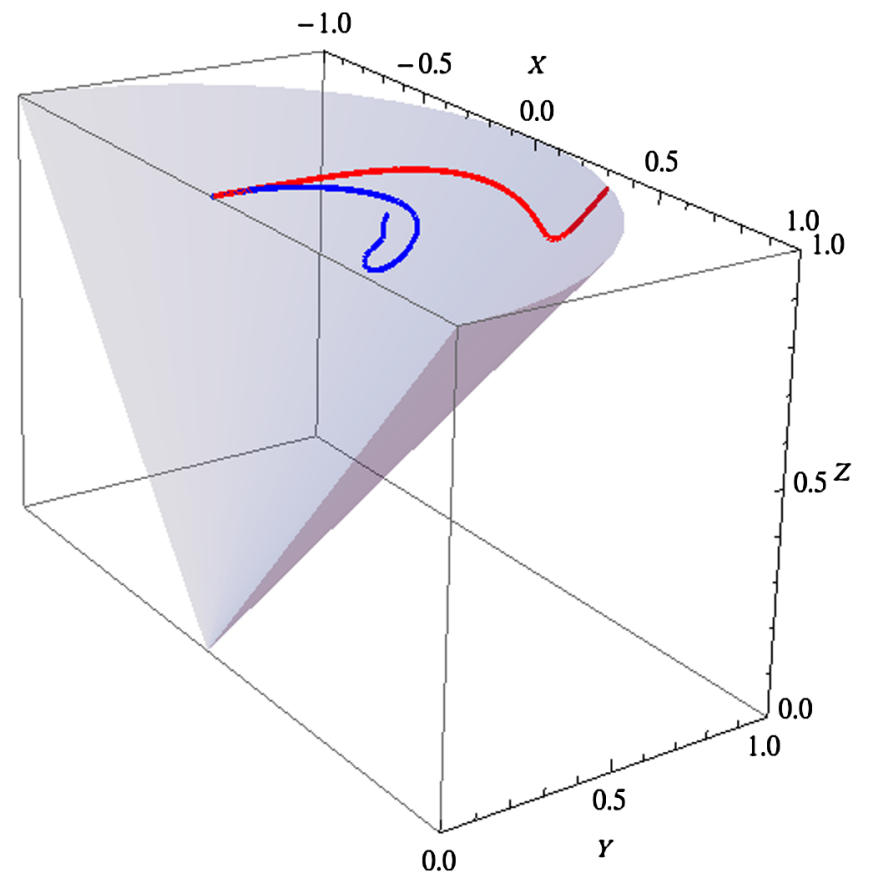

FIG. 2 (color online). The phase space trajectories for models with $\lambda=1$ (red) and $\lambda=4$ (blue). In each case $\beta=0.3, m_{0}=$ $\Lambda=H_{0}$ and the initial conditions are $\phi\left(N_{\mathrm{i}}\right)=1, \phi^{\prime}\left(N_{\mathrm{i}}\right)=0$. The initial values of $N_{\mathrm{i}}=\ln a_{\mathrm{i}}$ and $H\left(N_{\mathrm{i}}\right)$ were chosen such that the Universe begins in a matter dominated phase at redshift 10 with $\Omega_{\mathrm{m}}=0.99999$.

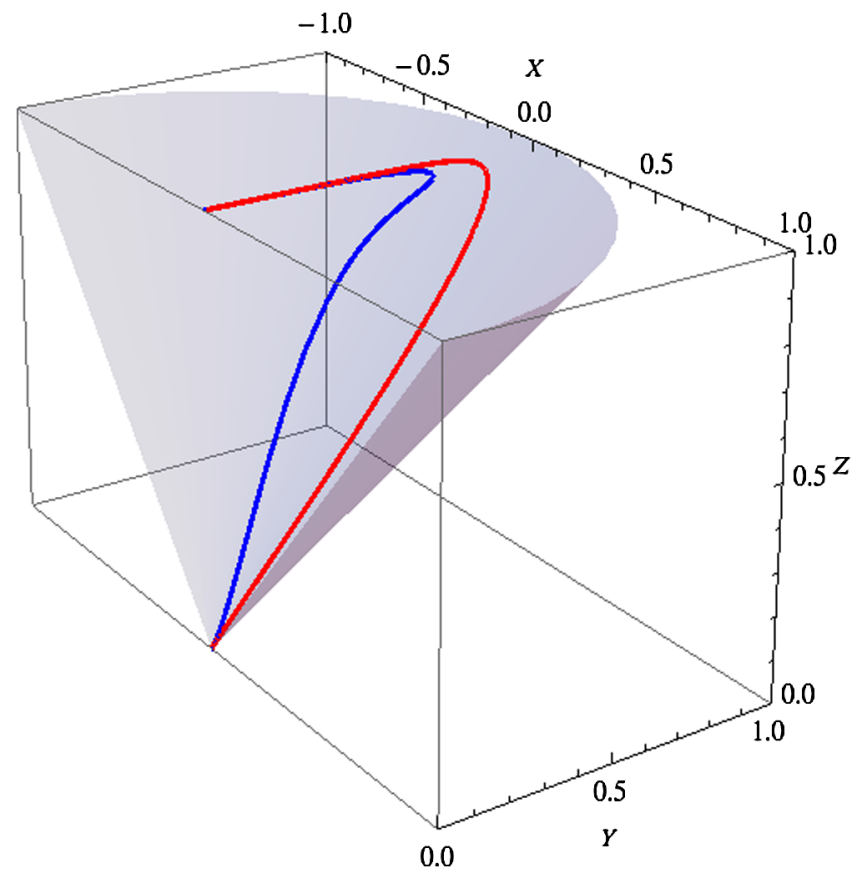

FIG. 3 (color online). The phase space trajectories for models with $\lambda=1$ (red) and $\lambda=4$ (blue). In each case $\beta=3$, $m_{0}=\Lambda=H_{0}$ and the initial conditions are those indicated in Fig. 2.
Einstein and Jordan frames are equivalent, and so are the coordinates used to parametrize the phase spaces.

\section{Fixed points when $\beta=\lambda / 2$}

As remarked above, the phase space is two dimensional when $\beta=\lambda / 2$. To see this, we can use Eq. (33) in the Friedmann constraint (19) to find

$$
1=\left(1+2 \mu^{2}\right) x^{2}+y^{2}+\Omega_{\mathrm{m}}\left(1+2 \mu^{2} \frac{x^{2}}{y^{2}}\right)^{\frac{3}{2}},
$$

with $\mu=m_{0} / \Lambda$. In this case, the phase space is elliptical. There are two routes by which one can proceed to analyze the fixed points of the system. The first is to reformulate the equations in terms of $x$ and $y$ and use the Friedmann equation to eliminate $\Omega_{\mathrm{m}}$. One can then find the fixed points of the two-dimensional system and proceed in the usual manner. The second is to continue to work in the three-dimensional framework and apply the constraint, which in our variables is [see Eq. (34)]

$$
Y^{2}=\frac{2 \mu^{2} X^{2}}{1-Z^{\frac{4}{3}}}
$$

Here, we adopt the second approach in order to make contact with the previous analysis. Substituting the constraint (40) into Eqs. (35)-(37) in order to eliminate $Y$, one finds the equations in the reduced phase space, which are given in Appendix B due to their length.

The resulting fixed points are given in Table III with the corresponding cosmological parameters given in Table IV. The eigenvalues are

TABLE III. The fixed points and lines when $\beta=\lambda / 2$.

\begin{tabular}{lcccc}
\hline \hline Name & $\mathrm{X}$ & $\mathrm{Y}$ & $\mathrm{Z}$ & Existence \\
\hline (1) & 0 & 0 & $0<Z \leq 1$ & All \\
(2) & 0 & 0 & 0 & All \\
(3) & 0 & 0 & 1 & All \\
(4) & $\sqrt{\frac{3}{2 \lambda^{2}}}\left(1-2 \mu^{2}\right)^{3 / 4}$ & $\sqrt{\frac{3}{2 \lambda^{2}}}\left(1-2 \mu^{2}\right)^{3 / 4}$ & $\left(1-2 \mu^{2}\right)^{3 / 4}$ & $\mu<\frac{1}{\sqrt{2}}$, \\
& & $\lambda \geq \sqrt{3}$ \\
(5) & $\frac{\lambda}{\sqrt{6}}\left(\frac{2 \lambda^{2} \mu^{2}}{\lambda^{2}-6}+1\right)^{3 / 4}$ & $\sqrt{1-\frac{\lambda^{2}}{6}}\left(\frac{2 \lambda^{2} \mu^{2}}{\lambda^{2}-6}+1\right)^{3 / 4}\left(\frac{2 \lambda^{2} \mu^{2}}{\lambda^{2}-6}+1\right)^{3 / 4}$ & $\lambda^{2}<\frac{6}{1+2 \mu^{2}}$ \\
\hline \hline
\end{tabular}

TABLE IV. The cosmological variables at the fixed points when $\beta=\lambda / 2$.

\begin{tabular}{lcccc}
\hline \hline Name & $H^{\prime} / H$ & $q$ & $w_{\text {eff }}$ & $\Omega_{\mathrm{DE}}$ \\
\hline$(1)$ & $-\frac{3}{2}$ & $\frac{1}{2}$ & 0 & $1-Z^{2}$ \\
$(2)$ & $-\frac{3}{2}$ & $\frac{1}{2}$ & 0 & 1 \\
$(3)$ & $-\frac{3}{2}$ & $\frac{1}{2}$ & 0 & 0 \\
$(4)$ & $-\frac{3}{2}$ & $\frac{1}{2}$ & $\frac{\lambda^{2}}{3}-1$ & $\left(\frac{3}{\lambda^{2}}-1\right)\left(1-2 \mu^{2}\right)^{3 / 2}+1$ \\
$(5)$ & $-\frac{\lambda^{2}}{2}$ & $\frac{\lambda^{2}}{2}-1$ & 0 & 1 \\
\hline \hline
\end{tabular}


(1) $e_{1}=\frac{3}{2}, \quad e_{2}=0$,

(2) $e_{1}=\frac{3}{2}, \quad e_{2}=0$,

(3) $e_{1}=\frac{3}{2}, \quad e_{2}=0$,

(4) $e_{ \pm}=-\frac{3}{4} \pm 3 \frac{\sqrt{\left(2\left(\lambda^{4}+18 \lambda^{2}-72\right) \mu^{2}+72-21 \lambda^{2}\right)}}{4 \lambda \sqrt{\left(2\left(\lambda^{2}-6\right) \mu^{2}+3\right)}}$,

(5) $e_{1}=\lambda^{2}-3, \quad e_{2}=-3+\frac{\lambda^{2}}{2}$.

One can see that the first three points are unstable and so we will ignore them from here on. The fourth point is a deformation of the stable spiral found when $\beta<\lambda / 2$ and when $\lambda>\sqrt{6}$. Its form is rather cumbersome but, by taking the limit $\lambda \rightarrow \infty$, one can see that the largest eigenvalue tends to zero from below and is therefore stable. ${ }^{11}$ The fifth point is a deformation of the stable attractor found when $\beta<\lambda / 2$ and when $\lambda<\sqrt{3}$. One can see that when $\mu>\sqrt{2}$ and $\lambda>\sqrt{3}$ the only stable point is the tip of the cone. Just like the analysis of the case $\beta \neq \lambda / 2$, this implies that the trajectories approach a center manifold at late times. Again, we will analyze this case by looking for an approximate late-time solution. The three possible types of solution are shown in Fig. 4.

\section{LATE-TIME SOLUTIONS}

In this section we address models that evolve towards the tip of the cone by looking for approximate late-time solutions. These were the cases $\beta>\lambda / 2$ and $\beta=\lambda / 2$.

\section{A. Solution when $\beta>\lambda / 2$}

At late times, one expects that the field has rolled down the potential sufficiently such that $\phi \gg 1$ and $\Omega_{\mathrm{m}} \ll 1$. Writing the Friedmann constraint (19) as

$3 H^{2}=\frac{\dot{\phi}^{2}}{2}\left(1+\frac{m_{0}^{2} e^{(2 \beta-\lambda) \phi}}{\Lambda^{2}}\right)+m_{0}^{2} e^{-\lambda \phi}+8 \pi G \rho u^{\frac{3}{2}}$

one can see that the final two terms are negligible compared with the term $3 H^{2}$ and so we have

$$
3 H^{2} \approx \frac{m_{0}^{2} \dot{\phi}^{2} e^{(2 \beta-\lambda) \phi}}{\Lambda^{2}} .
$$

Changing from coordinate time to $N=\ln a$ we have

\footnotetext{
${ }^{11}$ Whether or not it is an attractor or a stable spiral depends on the values of $\mu$ and $\lambda$.
}

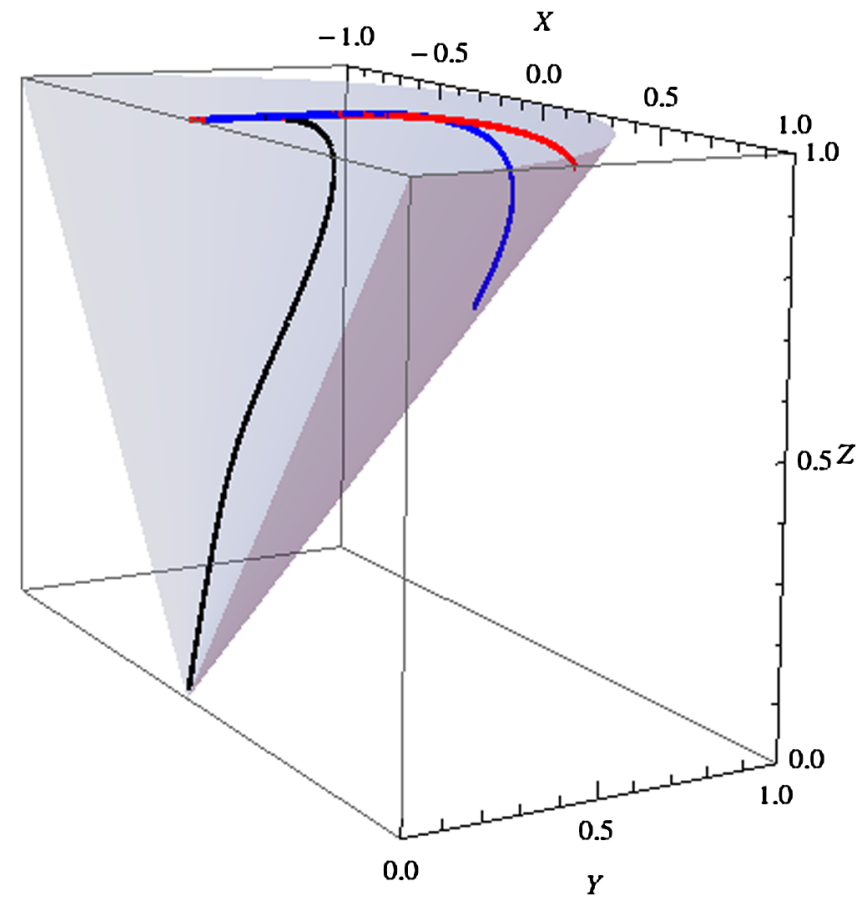

FIG. 4 (color online). The phase space trajectories for possible solutions when $\beta=\lambda / 2$. The blue line tends to fixed point (4) and corresponds to a model with $\mu=1, \lambda=1$. The red line tends to fixed point (5) and corresponds to a model with $\lambda=1, \mu=0.5$. The black line corresponds to a model with $\mu=1, \lambda=10$ and tends towards the tip of the cone. In each case $\Lambda=H_{0}$ and $m_{0}$ was fixed using the value of $\mu$. The initial conditions are those indicated in Fig. 2.

$$
\frac{m_{0}^{2} \phi^{\prime 2} e^{(2 \beta-\lambda) \phi}}{\Lambda^{2}}=3
$$

which is solved by

$$
\phi(N)=\frac{2}{2 \beta-\lambda} \ln \left(\sqrt{3} \frac{(2 \beta-\lambda)}{2} \frac{\Lambda}{m_{0}} N\right)
$$

This approximate solution is shown in Fig. 5 and one can see that it matches very closely with the numerical solution. Next, we can make the same approximations to Eq. (20) to find

$$
\frac{\dot{H}}{H^{2}}=-\frac{3}{2} \Omega_{\mathrm{m}} \frac{\dot{\phi}^{3}}{\Lambda^{3}} e^{3 \beta \phi}+\frac{1}{H}\left(\beta \dot{\phi}+\frac{\ddot{\phi}}{\dot{\phi}}\right),
$$

which, when written using $N$ as the time coordinate and applying the solution (49), becomes

$$
\frac{3}{2} \Omega_{\mathrm{m}} \phi^{\prime 3} \frac{H^{3}}{\lambda^{3}} e^{3 \beta \phi}=\frac{5 \beta-2}{2 N} .
$$

Taking the logarithm of both sides, differentiating with respect to $N$ and using the relation 


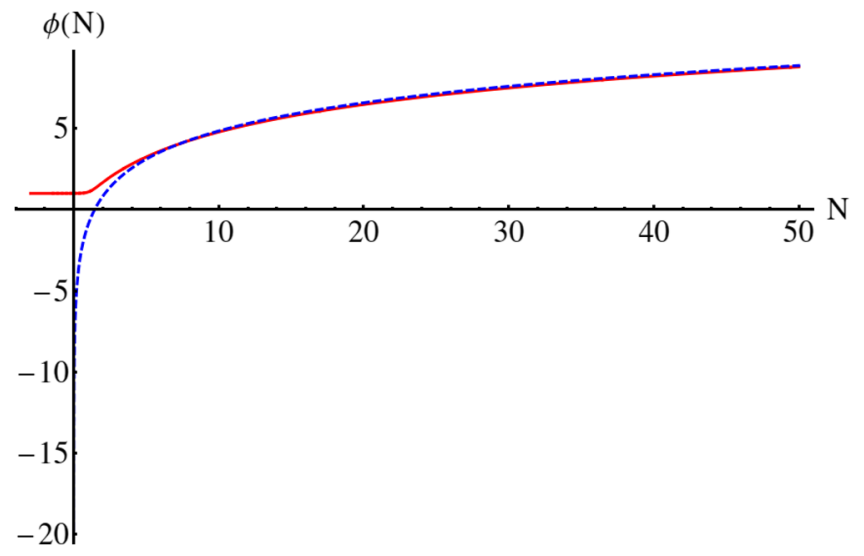

FIG. 5 (color online). The evolution of $\phi(N)$ found both numerically (red solid curve) and using the approximation (49) (blue dashed curve). The parameters used were $\beta=1.4$, $\lambda=2$ and $m_{0}=\Lambda=H_{0}$. The initial conditions are those indicated in Fig. 2.

$$
\frac{\Omega_{\mathrm{m}}{ }^{\prime}}{\Omega_{\mathrm{m}}}=-3-2 \frac{H^{\prime}}{H}
$$

we find

$$
\frac{H^{\prime}}{H}=3-\frac{2(\beta+\lambda)}{(2 \beta-\lambda) N} .
$$

This is plotted in Fig. 6 and one can again see that the approximation works very well at late times.

We can see that when $\beta>\lambda / 2$ the Universe will ultimately enter a phantom phase where $H^{\prime} / H$ tends to 3 , although many $e$-folds must elapse before the asymptotic value is reached. That being said, it is not necessarily the case that a large change in $N$ implies a large amount of

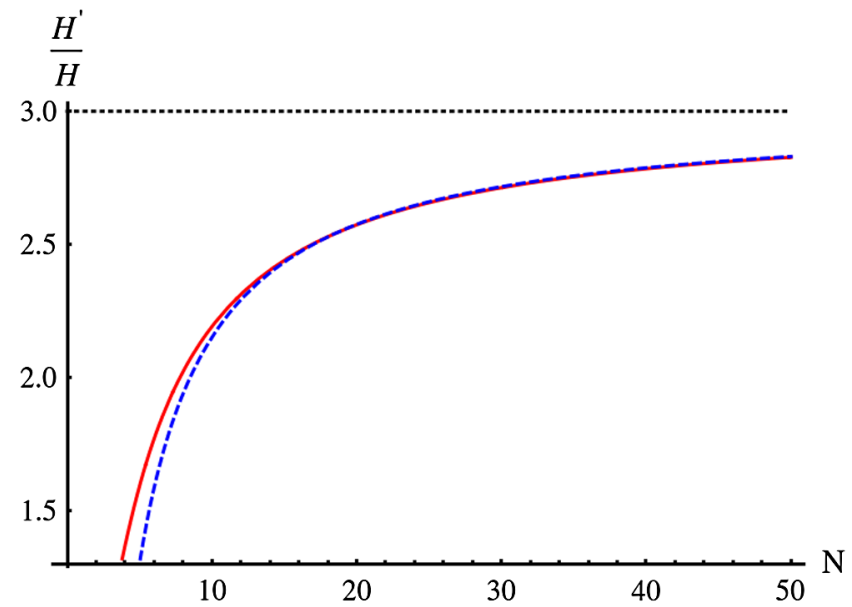

FIG. 6 (color online). The evolution of $H^{\prime} / H$ found both numerically (red solid curve) and using the approximation (49) (blue dashed curve). The asymptotic value of 3 is shown using the black dotted line. The parameters used were $\beta=1.4$, $\lambda=2$ and $m_{0}=\Lambda=H_{0}$. The initial conditions are those indicated in Fig. 2. coordinate time has elapsed. Indeed, recalling that $H=\mathrm{d} N / \mathrm{d} t$, the coordinate time is

$$
t(N)=\int_{N_{\mathrm{i}}}^{N} \frac{\mathrm{d} N^{\prime}}{H\left(N^{\prime}\right)} .
$$

Since the lapse is unity, this is the proper time for comoving observers. For nonphantom solutions such as the quintessencelike trajectories found in Sec. IV B, $H$ is a decreasing function of $N$ and so $t(N)$ is an exponentially increasing function. The phantom solutions, on the other hand, have $H(N)$ increasing exponentially and so $t(N)$ is a slowly evolving function at large $N$. Physically, this means that one expects a large number of $e$-folds in a short amount of proper time, and so the asymptotic phantom state is reached very quickly. This behaviour is plotted in Fig. 7 and can be understood by considering the Einstein frame. As the Universe expands, the field begins to roll and disformal effects become increasingly important. If the field does not begin to slow, the Jordan frame lapse approaches zero and little coordinate time evolves, despite the fact that the scale factor and field are evolving rapidly. When viewed in this manner, phantom behavior is a natural consequence of the disformal coupling.

We end this section by noting that the solution (53) implies that $q \approx-4$, or, equivalently, $w_{\text {eff }} \approx-3$. An equation of state this negative is in strong tension with observational data [56-60] but it is not necessarily the case that this value is reached at the present time. Indeed, examination of Fig. 6 reveals that, for the model studied there, the asymptotic value is not reached until far into the future. Whether a model predicts that the Universe is in the phantom phase at the present time or that it will undergo one at some point in the future depends on the initial conditions and model parameters such as $m_{0}$ and $\Lambda$, which do not determine the asymptotic state of the Universe but do control how quickly it is reached. For example, if one were to tune $m_{0} \gg H_{0}$ the field will begin to roll early on in the Universe's history and one would expect phantom behavior today. Conversely, tuning $m_{0} \ll H_{0}$ will result in the field being overdamped due to Hubble friction and the phantom behavior will only ensue far into the future. When fitting cosmological probes of the background expansion to data, Bayesian analysis will likely favor regions of parameter space where the phantom phase has not yet begun and so it is likely that cosmologically viable models can be found. Such an investigation would make an interesting topic for future work.

$$
\text { B. } \boldsymbol{\beta}=\lambda / 2
$$

When $\beta=\lambda / 2$ we can write the Friedmann equation as

$$
3 H^{2}=\left(1+2 \mu^{2}\right) \frac{\dot{\phi}^{2}}{2}+m_{0}^{2} e^{-\lambda \phi}+8 \pi G \rho u^{\frac{3}{2}} .
$$




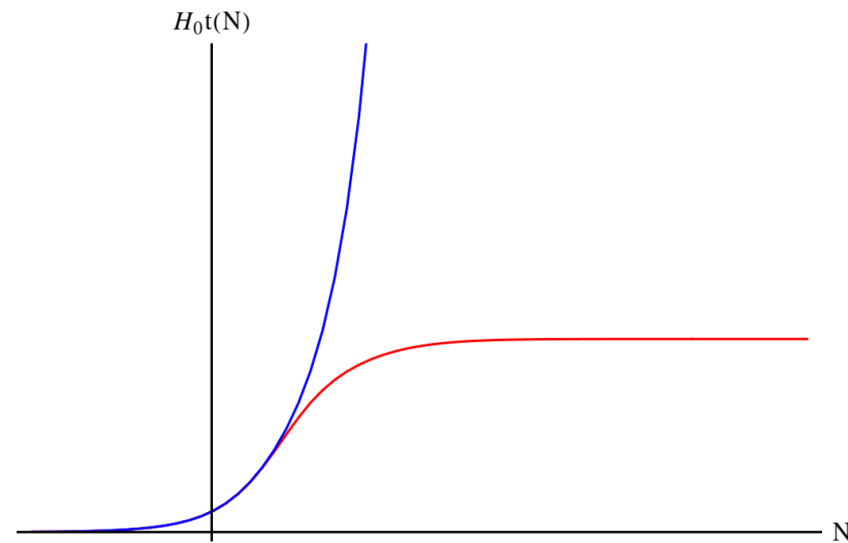

FIG. 7 (color online). The coordinate time as a function of $N$ for both quintessencelike solutions (blue) and phantom solutions (red). The parameters used were $\beta=1.4, \lambda=2$ (red) and $\beta=0.3, \lambda=2$ (blue). In both cases $m_{0}=\Lambda=H_{0}$. The initial conditions are those indicated in Fig. 2.

Again, one expects that $\phi \gg 1$ at late times but, unlike the previous case, there are no factors of $e^{2 \beta \phi}$ that become large in this limit. Instead, the second term is negligible and one has

$$
\begin{gathered}
1 \approx\left(1+2 \mu^{2}\right) \frac{\phi^{\prime 2}}{6}+\Omega_{\mathrm{m}} u^{\frac{3}{2}}, \quad \text { and } \\
0 \approx-\frac{\phi^{\prime 2}}{2}-\frac{3}{2} \Omega_{\mathrm{m}} u^{\frac{3}{2}}+\frac{\lambda}{2} \phi^{\prime},
\end{gathered}
$$

where the second equation comes from taking the limit $\phi \gg 1$ in Eq. (20). Unlike the previous case, it is not possible to find an exact analytic solution but one can find late-time scaling solutions by looking for solutions of the form $\phi^{\prime}=\delta_{1}, \Omega_{\mathrm{m}} u^{\frac{3}{2}}=\delta_{2}$. Under these assumptions, one is led to two equations for $\delta_{i}$ :

$$
\begin{gathered}
3=\frac{1}{2}\left(2 \mu^{2}+1\right) \delta_{1}^{2}+3 \delta_{2} \\
0=\frac{\lambda \delta_{2}}{2}-\frac{\delta_{1}^{2}}{2}-\frac{3 \delta_{2}}{2},
\end{gathered}
$$

which have the solutions

(1) $\delta_{1}=\frac{\sqrt{\lambda^{2}+12 \mu^{2}-6}+\lambda}{1-2 \mu^{2}}$

$$
\delta_{2}=\frac{2}{1-2 \mu^{2}}-\frac{\lambda\left(2 \mu^{2}+1\right)\left(\lambda-\sqrt{\lambda^{2}+12 \mu^{2}-6}\right)}{3\left(1-2 \mu^{2}\right)^{2}}
$$

(2) $\delta_{1}=\frac{6}{\sqrt{\lambda^{2}+12 \mu^{2}-6}+\lambda}$

$$
\delta_{2}=\frac{2}{1-2 \mu^{2}}-\frac{\lambda\left(2 \mu^{2}+1\right)\left(\lambda+\sqrt{\lambda^{2}+12 \mu^{2}-6}\right)}{3\left(1-2 \mu^{2}\right)^{2}} \text {. }
$$

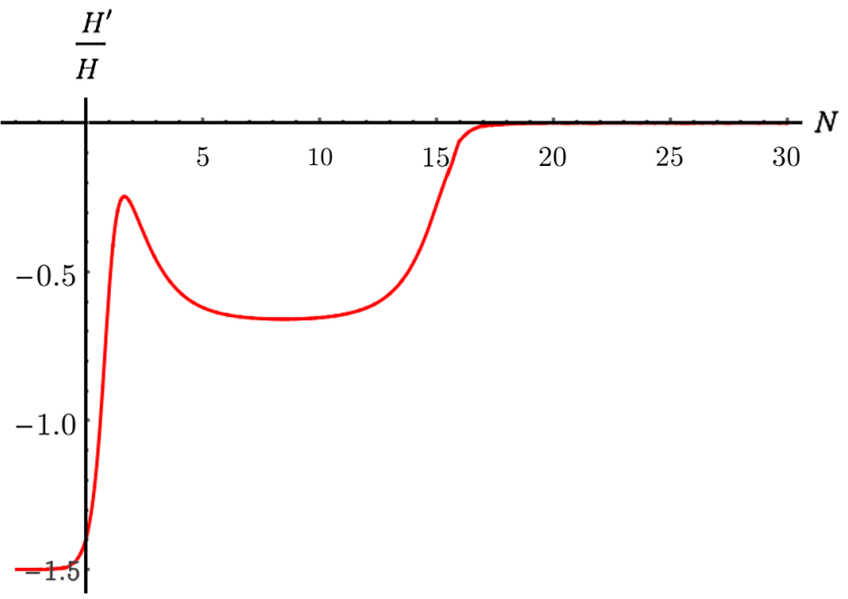

FIG. 8 (color online). $\quad H^{\prime} / H$ as a function of $N$ for a model with $\mu=1, m_{0}=H_{0}, \lambda=\sqrt{2}$ and $\Lambda=H_{0}$. The initial conditions are those indicated in Fig. 2.

Note that one requires $2 \mu^{2}>1$ in order for this type of solution to exist. If the converse is true the solution tends to fixed point (4) found in Sec. IV C. Given this constraint, one can see that solution (1) is incompatible with our assumption that $\phi \gg 1$ because $\phi^{\prime}=\delta_{1}<0$ and so only solution (2) is viable. A scaling relation such as this implies a definite prediction for the asymptotic state of the Universe. Indeed, since $\Omega_{\mathrm{m}} u^{\frac{3}{2}}$ is constant one has, using Eq. (52),

$$
\frac{H^{\prime}}{H}=3-\frac{3 \lambda}{2} \delta_{1},
$$

which implies

$$
\begin{gathered}
q=\frac{9 \lambda}{\sqrt{\lambda^{2}+12 \mu^{2}-6}+\lambda}-4, \quad \text { and } \\
w_{\text {eff }}=\frac{6 \lambda}{\sqrt{\lambda^{2}+12 \mu^{2}-6}+\lambda}-3 .
\end{gathered}
$$

One can see that in this case the asymptotic state of the Universe is a function of $\lambda$ and $\mu$. Note that since $\delta_{1}>0$, the Universe cannot accelerate with $H^{\prime} / H>3$. A natural question is whether it is possible for the Universe to achieve an asymptotic de Sitter state? Setting the left-hand side of (62) equal to zero and using (61) one finds this is achieved when

$$
\lambda=\sqrt{2} \sqrt{2 \mu^{2}-1} .
$$

As an example, we plot the evolution of $H^{\prime} / H$ as a function of $N$ for the case $\mu=1(\lambda=\sqrt{2})$ in Fig. 8. One can see that the Universe does indeed tend to a de Sitter phase at late times. 


\section{DISCUSSION AND CONCLUSIONS}

This paper has presented and studied the Jordan frame formulation of disformal gravity theories for the first time. The Einstein frame has been studied extensively and motivates this study for several reasons. First, there is an apparent metric singularity that previous studies have found, both numerically and analytically, to be approached on cosmological scales when calculating using the Einstein frame formulation. This result has some pathological implications but, as discussed in Sec. II, it is currently unknown whether or not is it a physical pathology or merely an artifact of working in the Einstein frame. This paper has taken the first steps towards answering this by studying the Jordan frame cosmology and looking for equivalent pathologies. Second, disformal transformations from the Einstein to Jordan frame do not preserve the lapse. This has the result that the proper time for observers in the Jordan frame is not aligned with the coordinate time, which makes the interpretation of Einstein frame calculations difficult from a technical point of view. The Jordan frame does not have this problem since the lapse is unity from the outset.

The first part of the paper was dedicated to analyzing the phase space of solutions using a dynamical systems analysis. We were successful in compactifying the three-dimensional phase space so that all solutions lie inside of the half-cone shown in Fig. 1. Interestingly, the phase space of the equivalent quintessence model (found by turning off the disformal couplings) coincides with the base of the cone, which allowed for transparent comparisons with quintessence. In particular, any trajectory that terminates on the base of the cone has a late-time cosmology that is indistinguishable from quintessence, at least at the background level. The fixed points on the base of the cone correspond to those found in the Einstein frame by previous studies precisely because disformal effects are absent and the time variables used to describe the dynamics in both frames are identical. Trajectories at the tip of the cone yield no information about the late-time cosmology and it was necessary to find approximate late-time solutions in order to discern the asymptotic state of the Universe. In this case, one can only relate the Einstein and Jordan frame time variables by integrating a nonlinear relation, and it is here that the power of the Jordan frame formalism becomes apparent.

The cosmological behavior can be summarized concisely in the $\beta-\lambda$ plane shown in Fig. 9. When $\beta<\lambda / 2$, all of the fixed points lie in the quintessence plane and so the latetime fixed points are identical to those found in [48], although their stability is different due to the phase space being three instead of two dimensional. When $\beta>\lambda / 2$, the only stable fixed point lies at the tip of the cone and so it was necessary to look for approximate late-time solutions. These were found in Sec. V where we showed that the Universe asymptotes to a phantom state where $w_{\text {eff }}=-3$ $\left(\dot{H} / H^{2}=3\right)$ independent of the model parameters. One can see from the various figures that the pathological behavior

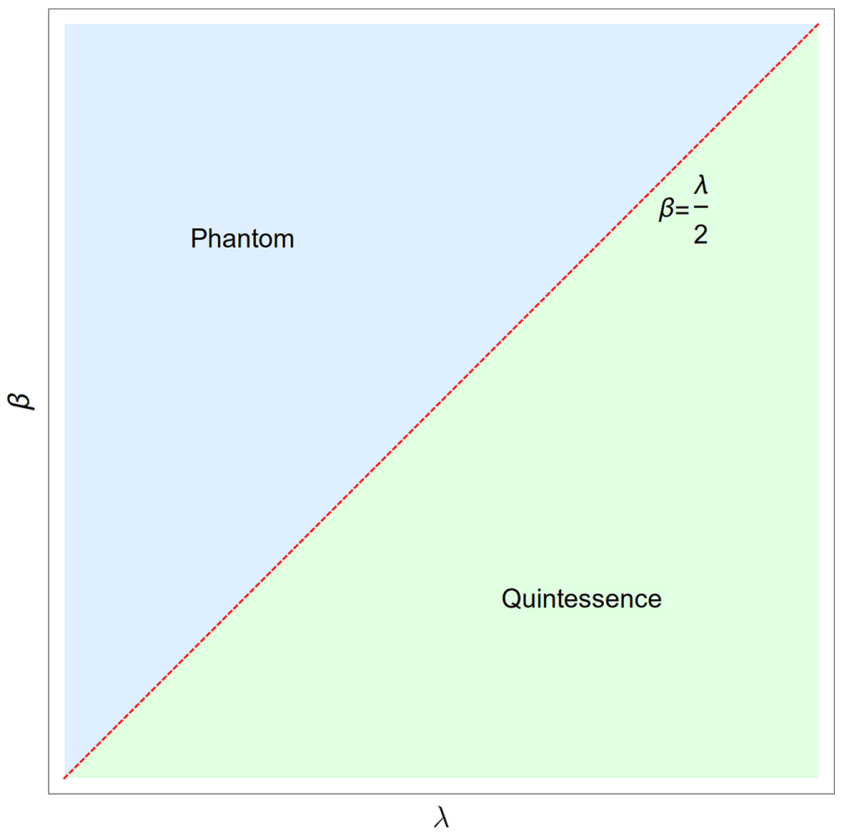

FIG. 9 (color online). The cosmological solutions found in this work.

is typically reached in the future for universes that start from matter domination and so it may be possible to reconcile the models with current observations. In particular, there are several model parameters, such as $m_{0}$ and $\Lambda$, that do not alter the position of the fixed points or the stability. One would therefore expect a wide region in parameter space where the Universe is close to $\Lambda \mathrm{CDM}$ today but may undergo a phantom phase sometime in the future. Such a model is not at odds with current observations. When fitting the model to cosmological probes of the background cosmology, it is likely that this region will be preferred by Bayesian fitting methods, although such analyses lie beyond the scope of this work.

There is a marginal case given by $\beta=\lambda / 2$ where the phase space is reduced to 2 . In this case we found two late-time attracting fixed points that lie inside the cone and one that lies at the tip. By looking for late-time scaling solutions we derived the asymptotic value of $\dot{H} / H^{2}$ for solutions that approach the tip and, in particular, were able to show that by tuning the parameters, a late-time de Sitter phase can be reached.

This solution deserves further comment in light of the cosmological constant problem. In order to achieve the solution required for an asymptotic de Sitter phase it was necessary to tune $\beta=\lambda / 2$ but this is not enough. One must further tune $\lambda$ and $\mu$ to values where the fixed point at the tip is the only stable one and the asymptotic value of $w_{\text {eff }}$ is exactly -1 . The theory does not contain any sort of protective symmetry and thus the tunings required are unlikely to be technically natural. Furthermore, the model has nothing to say about the old cosmological constant problem because we have set all contributions to the 
cosmological constant from both the scalar and matter sectors to zero from the outset. Given this, the asymptotically de Sitter cosmological solution found here has little to say about the cosmological constant problem, and the finetuned model is hardly a compelling alternative to $\Lambda \mathrm{CDM}$.

One of the goals of this paper is to discuss the metric singularity found by previous works using the Einstein frame formulation of the theory. The pathologies associated with this singularity were discussed at length in Sec. II. There, we noted that it is a coordinate singularity since one can find a gauge where the metric is perfectly regular and that it is apparently absent in the Jordan frame since one can work in this gauge from the outset. We showed that the singularity is located at the tip of the cone in this gauge and, furthermore, that trajectories approaching the tip are those that exhibit late-time phantom behavior. The physical manifestation of the singularity is then clear: the Universe undergoes phantom behavior à la [61]. Retrospectively, this is somewhat to be expected from the Einstein frame behavior: the approach to the singularity corresponds to the Jordan frame lapse approaching zero so that the clock for comoving observers slows down. A large number of $e$-folds can then pass in a short amount of time, which is precisely the behavior of a phantom Universe.

We end by discussing the generality of our findings. In particular, the choice among a theory that is identical to quintessence, a phantom Universe or a finely tuned de Sitter phase seems unappealing compared with simpler models. Here, we have only considered models where the scalar potential and disformal factor are exponential. This choice was made in order to yield the minimal phase space and preserve some of the scaling symmetry present in quintessence models. More general models will have a larger phase space that will require different variables to explore and one hence expects a new set of fixed points. Despite this, one would expect the qualitative features we have found here to apply. In particular, the fixed points were found to correspond to either phantom behavior or the equivalent quintessence model except for a finely tuned set of parameters. When written in terms of the cosmological variables and using $N=\ln a$ as a proxy for time in the Einstein frame, the disformal coupling leaves the spatial component unchanged but the Jordan frame lapse is given by $N^{2}=1-B(\phi)^{2} H^{2} \phi^{2} / \Lambda^{2}$. All nonphantom Universes have $H \rightarrow 0$ at late times and so one expects a set of fixed points corresponding to the equivalent quintessence models precisely because the disformal coupling is set to zero dynamically and the Jordan and Einstein frames are equivalent. One can then discern the requirements for the existence of new fixed points corresponding to nonzero disformal couplings: either the Einstein frame Universe must be phantom so that $H$ or $\phi^{\prime}$ increases without bound, or $B(\phi)$ must be chosen such that it is a strongly increasing function of $\phi$. This was the case with exponential models. Indeed, here we found that the disformal factor was only nonzero for values of $\beta$ that were large enough to compensate for the decreasing of $H$. One can then see that phantom behavior is expected for any model where $B(\phi)$ can increase rapidly enough at late times, what is not universal is the prediction that $w_{\text {eff }}=-3$, which is likely to be a theory-dependent prediction. Said another way, one can design models that do not exhibit phantom behavior by construction. One simple example of this is simply $B=1$, which shows only quintessence fixed points. A more general example is the case of monomial potentials $V(\phi) \sim \phi^{n}, B(\phi) \sim \phi^{m}$ (with $n$ and $m$ positive even integers). In this case, one would expect $\phi$ to roll to the minimum of the potential located at $\phi=0$ at late times so that $B(\phi)$ tends to zero and the system behaves like quintessence. In light of the discussion above, we conclude that the general features found here-quintessencelike fixed points and phantom behavior-are properties of more general disformal dark energy models.

In order to find fixed points that were neither quintessence models nor phantom Universes it was necessary to fine-tune several model parameters to specific values. This corresponded to reducing the dimension of the phase space so that $B H / \Lambda$ was fixed by the kinetic and potential energy. In this case it could neither grow without bound nor become zero. Such a fine-tuning is a very special property of the model considered here and it is unlikely to be a feature of more general models. More technically, the symmetries of the equations of motion $\left[\dot{\phi}^{2} \sim H^{2}, V(\phi) \sim\right.$ $H^{2}$ and $V_{\phi} \sim V(\phi)$ ] were crucial in allowing one to have the minimal possible phase space dimension and to identify the requisite parameter tunings. Constructing other theories that exhibit these features would require looking at the symmetries present when the disformal coupling is absent and choosing the functional form of $B(\phi)$ appropriately such that the dimension of the phase space can be preserved with suitable parameter tunings. It is then clear that the novel fixed points found in the marginal case are not general and require finely tuned simple models to exist.

We have not included a conformal factor in our analysis and it is unlikely that this will have any mitigating effects for the pathologies. Indeed, a conformal factor was included in the Einstein frame analysis of [34] with the only effect being to move the location of the fixed points. Such factors are strongly constrained by solar system tests and so the change is expected to be minimal. Indeed, if a conformal factor $A^{2}(\phi)$ is present then the Cassini constraint on the PPN parameter $\gamma$ [62] constrains $\mathrm{d} \ln A / \mathrm{d} \phi<$ $10^{-3}[42,63]$, which is the factor that appears in the equations governing the cosmological dynamics.

Finally, one can relax the universal coupling and couple to dark matter only, at the cost of introducing violations of the equivalence principle, which are poorly constrained in the dark sector. In this case, the relevant fixed points for observers are those found in [34], although the metric governing the motion of dark matter is that of a phantom universe and so one expects a drastic suppression of latetime structure compared with GR. 


\section{ACKNOWLEDGMENTS}

We would like to thank Jack Morrice, Cornelius Ramph, Fabian Schmidt, and Thomas Tram for several enlightening conversations. We are grateful to the SEPnet summer placement scheme for its support in organizing the placement in which S. V. has participated, and to the University of Portsmouth for hosting S. V. during the time this work was carried out.

\section{APPENDIX A: TRANSFORMATION TO THE JORDAN FRAME}

In this appendix we transform the Einstein frame action to the Jordan frame and derive the Friedmann and Klein-Gordon equations.

\section{The Jordan frame action}

Our starting point is the Einstein frame action (3), which we write as

$$
\begin{aligned}
S & =\int \mathrm{d}^{4} x M_{\mathrm{pl}}{ }^{2}\left[\mathcal{L}_{g}+\mathcal{L}_{\phi}\right]+S_{\mathrm{m}}[\tilde{g}] \quad \text { with } \\
\mathcal{L}_{\mathrm{g}} & =\frac{\sqrt{-g} R(g)}{2} \text { and } \\
\mathcal{L}_{\phi} & =\sqrt{-g}\left[-\frac{1}{2} \tilde{\nabla}_{\mu} \phi \tilde{\nabla}^{\mu} \phi-V(\phi)\right] .
\end{aligned}
$$

Bettoni and Liberati [14] have shown that the Horndeski action [24] - the most general scalar-tensor theory with manifestly second-order field equations - is invariant under disformal transformations and furthermore that the Einstein frame exists only when terms quintic in the scalar are absent. For this reason, we expect that the Jordan frame action takes the form

$$
\begin{aligned}
S= & \int \mathrm{d}^{4} x \sqrt{-\tilde{g}}\left(G_{2}(\phi, X)+G_{3}(\phi, X) \square \phi+G_{4}(\phi, X) R(\tilde{g})\right. \\
& \left.+G_{4, X}\left[(\square \phi)^{2}-\tilde{\nabla}_{\mu} \tilde{\nabla}_{\nu} \phi \tilde{\nabla}^{\mu} \tilde{\nabla}^{\nu} \phi\right]\right)+S_{\mathrm{m}}\left[\tilde{g}_{\mu \nu}\right], \quad \text { (A2) }
\end{aligned}
$$

where $G_{i}$ are arbitrary functions, $X=-\tilde{g}^{\mu \nu} \partial_{\mu} \phi \partial_{\nu} \phi / 2$, and $\square=\tilde{g}^{\mu \nu} \nabla_{\mu} \nabla_{\nu}$. Our strategy is then to transform each term in (A1) into the Jordan frame by inverting (4) and then performing manipulations to get it into the form (A2). To accomplish this, we follow the methods of $[15,27]$. We begin by inverting (4) to find

$$
\begin{gathered}
g_{\mu \nu}=\tilde{g}_{\mu \nu}-\frac{B^{2}(\phi)}{\Lambda^{2}} \partial_{\mu} \phi \partial_{\nu} \phi \quad \text { and } \\
g^{\mu \nu}=\tilde{g}^{\mu \nu}+\frac{B^{2}(\phi)}{\Lambda^{2}} \frac{\tilde{\nabla}^{\mu} \phi \tilde{\nabla}^{\nu} \phi}{1+\frac{2 B^{2}(\phi) X}{\Lambda^{2}}},
\end{gathered}
$$

where all contractions are performed using $\tilde{g}_{\mu \nu}$. Next, we introduce the tensor

$$
\begin{aligned}
\mathcal{K}_{\mu \nu}^{\alpha} & =\Gamma_{\mu \nu}^{\alpha}-\tilde{\Gamma}_{\mu \nu}^{\alpha} \\
= & \frac{B^{2}(\phi) \tilde{\nabla}^{\alpha} \phi \tilde{\nabla}_{\mu} \tilde{\nabla}_{\nu} \phi+B(\phi) B_{\phi}(\phi) \tilde{\nabla}^{\alpha} \phi \partial_{\mu} \phi \partial_{\nu} \phi}{\Lambda^{2}\left(1+\frac{2 B^{2}(\phi) X}{\Lambda^{2}}\right)} .
\end{aligned}
$$

Using the identity $2 \tilde{\nabla}_{[\mu} \tilde{\nabla}_{\nu]} v^{\beta}=R_{\beta \mu \nu}^{\alpha} v^{\beta}$ one finds [15]

$$
R_{\beta \mu \nu}^{\alpha}=\tilde{R}_{\beta \mu \nu}^{\alpha}+2 \tilde{\nabla}_{[\mu} \mathcal{K}_{\nu] \beta}^{\alpha}+2 \mathcal{K}_{\sigma[\mu}^{\alpha} \mathcal{K}_{\nu] \beta}^{\alpha},
$$

which, after making the appropriate contractions and using Eqs. (6) and (A3), can be used to transform $\mathcal{L}_{g}$ :

$$
\begin{aligned}
\frac{\mathcal{L}_{g}}{\sqrt{-\tilde{g}}}= & \frac{\sqrt{-g}}{\sqrt{-\tilde{g}}}\left[g^{\mu \nu}\left(\tilde{R}^{\alpha}{ }_{\mu \alpha \nu}-2 \mathcal{K}^{\alpha}{ }_{\sigma[\alpha} \mathcal{K}^{\sigma}{ }_{\mu] \nu}\right)\right] \\
= & \sqrt{1+\frac{2 B^{2}(\phi) X}{\Lambda^{2}}} \frac{\tilde{R}}{2}+\frac{B^{2}(\phi)}{\Lambda^{2} \sqrt{1+\frac{2 B^{2}(\phi) X}{\Lambda^{2}}}} \tilde{R}_{\mu \nu} \tilde{\nabla}^{\mu} \phi \tilde{\nabla}^{\nu} \phi \\
& -\frac{B^{2}(\phi)}{2 \Lambda^{2} \sqrt{1+\frac{2 B^{2}(\phi) X}{\Lambda^{2}}}}\left[(\square \phi)^{2}-\tilde{\nabla}_{\mu} \tilde{\nabla}_{\nu} \phi \tilde{\nabla}^{\mu} \tilde{\nabla}^{\nu} \phi\right]+\frac{B(\phi) B_{\phi}(\phi)}{\Lambda^{2}\left(1+\frac{2 B^{2}(\phi) X}{\Lambda^{2}}\right)^{\frac{3}{2}}}\left[\tilde{\nabla}^{\mu} \phi \tilde{\nabla}_{\mu} \tilde{\nabla}_{\nu} \phi \tilde{\nabla}^{\nu} \phi+2 X \square \phi\right] \\
& +\frac{B^{4}(\phi)}{\Lambda^{2}\left(1+\frac{2 B^{2}(\phi) X}{\Lambda^{2}}\right)^{\frac{3}{2}}}\left[\square \phi \tilde{\nabla}^{\mu} \phi \tilde{\nabla}_{\mu} \tilde{\nabla}_{\nu} \phi \tilde{\nabla}^{\nu} \phi+\tilde{\nabla}^{\mu} \phi \tilde{\nabla}^{\nu} \phi \tilde{\nabla}_{\alpha} \tilde{\nabla}_{\nu} \phi \tilde{\nabla}^{\alpha} \tilde{\nabla}_{\mu} \phi\right] .
\end{aligned}
$$

This is not yet in Horndeski form; there are two quartic terms and one cubic term that need to be removed. Furthermore, there is a term proportional to $\tilde{R}_{\mu \nu} \tilde{\nabla}^{\mu} \phi \tilde{\nabla}^{\nu} \phi$. We can remove this term and the quartic one by adding a total derivative of the form $\tilde{\nabla}_{\mu} \xi^{\mu}$ with

$$
\xi^{\mu}=\frac{B^{2}(\phi)}{2 \Lambda^{2} \sqrt{1+\frac{2 B^{2}(\phi) X}{\Lambda^{2}}}}\left[\tilde{\nabla}^{\mu} \phi \square \phi-\tilde{\nabla}^{\mu} \tilde{\nabla}^{\nu} \phi \partial_{\nu} \phi\right] .
$$

This simplifies the action to 


$$
\frac{\mathcal{L}_{g}}{\sqrt{-\tilde{g}}}=\sqrt{1+\frac{2 B^{2}(\phi) X}{\Lambda^{2}}} \frac{\tilde{R}}{2}+\frac{B^{2}(\phi)}{2 \Lambda^{2} \sqrt{1+\frac{2 B^{2}(\phi) X}{\Lambda^{2}}}}\left[(\square \phi)^{2}-\tilde{\nabla}_{\mu} \tilde{\nabla}_{\nu} \phi \tilde{\nabla}^{\mu} \tilde{\nabla}^{\nu} \phi\right]-\frac{B(\phi) B_{\phi}(\phi)}{\Lambda^{2} \sqrt{1+\frac{2 B^{2}(\phi) X}{\Lambda^{2}}}}\left[2 X \square \phi+\tilde{\nabla}^{\mu} \phi \tilde{\nabla}_{\mu} \tilde{\nabla}_{\nu} \phi \tilde{\nabla}^{\nu} \phi\right] .
$$

One can see that the quartic terms are in Horndeski form but there is still one cubic term that does not fit. This too can be removed by subtracting a second total derivative $\tilde{\nabla}_{\mu} \zeta^{\mu}$ with

$$
\zeta^{\mu}=\sqrt{1+\frac{2 B^{2}(\phi) X}{\Lambda^{2}}} \frac{B_{\phi}(\phi)}{B(\phi)} \tilde{\nabla}^{\mu} \phi .
$$

The action then becomes

$$
\begin{aligned}
\frac{\mathcal{L}_{g}}{\sqrt{-\tilde{g}}}= & \sqrt{1+\frac{2 B^{2}(\phi) X}{\Lambda^{2}}} \frac{\tilde{R}}{2}+\frac{B^{2}(\phi)}{2 \Lambda^{2} \sqrt{1+\frac{2 B^{2}(\phi) X}{\Lambda^{2}}}}\left[(\square \phi)^{2}-\tilde{\nabla}_{\mu} \tilde{\nabla}_{\nu} \phi \tilde{\nabla}^{\mu} \tilde{\nabla}^{\nu} \phi\right]-\frac{B_{\phi}(\phi)}{B(\phi) \Lambda^{2} \sqrt{1+\frac{2 B^{2}(\phi) X}{\Lambda^{2}}}}\left[1+\frac{4 B^{2}(\phi) X}{\Lambda^{2}}\right] \square \phi \\
& +2 X\left[\frac{B_{\phi \phi}}{B^{2}(\phi)} \sqrt{1+\frac{2 B^{2}(\phi) X}{\Lambda^{2}}}-\frac{B_{\phi}^{2}(\phi)}{B^{2}(\phi) \sqrt{1+\frac{2 B^{2}(\phi) X}{\Lambda^{2}}}}\right]
\end{aligned}
$$

which is now in the Horndeski form.

Next, we need to transform $\mathcal{L}_{\phi}$. This is a lot simpler since one only needs to transform the metric determinant and the metric appearing in the kinetic term using (A4) to find

$\frac{\mathcal{L}_{\phi}}{\sqrt{-\tilde{g}}}=\sqrt{1+\frac{2 B^{2}(\phi) X}{\Lambda^{2}}}\left[X-\frac{2 B^{2}(\phi) X^{2}}{\Lambda^{4}\left(1+\frac{2 B^{2}(\phi) X}{\Lambda^{2}}\right)}-V(\phi)\right]$.

The action is then in Horndeski form with

$$
\begin{gathered}
G_{4}(\phi, X)=\frac{M_{\mathrm{pl}}^{2}}{2} \sqrt{1+\frac{2 B^{2}(\phi) X}{\Lambda^{2}}} \\
G_{3}(\phi, X)=-M_{\mathrm{pl}}^{2} \frac{B_{\phi}(\phi)}{B(\phi) \Lambda^{2} \sqrt{1+\frac{2 B^{2}(\phi) X}{\Lambda^{2}}}}\left[1+\frac{4 B^{2}(\phi) X}{\Lambda^{2}}\right] \\
G_{2}(\phi, X)=M_{\mathrm{pl}^{2}} \sqrt{1+\frac{2 B^{2}(\phi) X}{\Lambda^{2}}}\left[\frac{2 X B_{\phi \phi}}{B^{2}(\phi)}\right. \\
-\frac{2 X B_{\phi}^{2}(\phi)}{B^{2}(\phi)\left(1+\frac{2 B^{2}(\phi) X}{\Lambda^{2}}\right)}+X \\
\left.-\frac{2 B^{2}(\phi) X^{2}}{\Lambda^{4}\left(1+\frac{2 B^{2}(\phi) X}{\Lambda^{2}}\right)}-V(\phi)\right] .
\end{gathered}
$$

Note that a similar action was obtained in [64].

\section{The field equations}

Given Eqs. (A14)-(A16), it is clear that the resulting field equations will be cumbersome and complicated. Since we are only interested in the homogeneous and isotropic Friedmann equations it is simplest to first reduce the action to minisuperspace using the coordinates

$$
\mathrm{d} \tilde{s}^{2}=-N^{2}(t) \mathrm{d} t^{2}+a(t)^{2} \mathrm{~d} \vec{x}^{2} ; \quad \phi=\phi(t) .
$$

The Friedmann and Klein-Gordon equations can then be found using the Euler-Lagrange equations for $N(t), a(t)$ and $\phi(t)$ and setting $N(t)=1$. Setting $B(\phi)=e^{\beta \phi^{12}}$ one finds

$$
\begin{aligned}
S[N(t), a(t), \phi(t)]= & \int \mathrm{d} t \frac{a(t)^{3}}{\sqrt{1+\frac{e^{2 \beta \phi}}{N^{2} \Lambda^{2}}}}\left[3 \gamma \frac{\dot{a}^{2}}{a^{2} N^{2}}-3 \gamma \frac{\dot{a} \dot{N}}{a N^{2}}\right. \\
& +3 \gamma \beta \frac{\dot{a} \dot{\phi}}{a N}-\beta \gamma \frac{\dot{N} \dot{\phi}}{N^{2}}+\frac{\dot{\phi}^{2}}{2} \\
& -V(\phi) N^{2}\left(1+\frac{e^{2 \beta \phi}}{N^{2} \Lambda^{2}}\right)+\beta^{2} \frac{e^{2 \beta \phi} \dot{\phi}^{4}}{\Lambda^{2} N^{3}} \\
& +3 \frac{\ddot{a}}{a N}\left(1+\frac{e^{2 \beta \phi}}{N^{2} \Lambda^{2}}\right) \\
& \left.+\beta \gamma \frac{\ddot{\phi}}{N}+3 \frac{e^{2 \beta \phi} \dot{a} \dot{\phi} \ddot{\phi}}{\Lambda^{2} a N^{3}}\right],
\end{aligned}
$$

\footnotetext{
${ }^{12}$ We do this for simplicity; it is not necessary to specialize at this stage but leaving the function general results in a far longer expression.
} 
where

$$
\gamma=1+2 \frac{e^{2 \beta \phi}}{N^{2} \Lambda^{2}}
$$

The Euler-Lagrange equation for $N(t)$ yields the Friedmann equation (19) (after setting $N=1$ ), which can be used in the Euler-Lagrange equations for $a(t)$ and $\phi(t)$ to find Eqs. (20) and (22).

\section{APPENDIX B: DYNAMICAL SYSTEM WHEN $\beta=\lambda / 2$}

In this appendix we present the dynamical system after substituting the constraint (40) into (35)-(37) to eliminate $Y$. They are

$$
\begin{aligned}
& U_{1} \frac{\mathrm{d} X}{\mathrm{~d} N}=-X\left(3 X^{4}\left(4\left(\mu^{2}-1\right) Z^{\frac{4}{3}}+3 Z^{8 / 3}+1-4 \mu^{4}\right)+2 \sqrt{6} \lambda X^{3} Z\left(2 \mu^{2}-2\left(2 \mu^{2}+1\right) Z^{4}+Z^{8 / 3}+1\right)\right. \\
& \left.-6 X^{2} Z^{2}\left(Z^{\frac{4}{3}}-1\right)\left(2 \mu^{2}+4 Z^{\frac{4}{3}}-3\right)+\sqrt{6} \lambda X Z^{3}\left(Z^{\frac{4}{3}}-1\right)^{2}-3 Z^{4}\left(Z^{\frac{4}{3}}-1\right)^{2}\right), \\
& U_{2} \frac{\mathrm{d} Y}{\mathrm{~d} N}=\mu X\left(3 X^{4}\left(2 \mu^{2}+Z^{\frac{4}{3}}-1\right)\left(2 \mu^{2}-3 Z^{\frac{4}{3}}+1\right)+2 \sqrt{6} \lambda\left(2 \mu^{2}+1\right) X^{3} Z\left(Z^{\frac{4}{3}}-1\right)\right. \\
& \left.+6 X^{2} Z^{2}\left(Z^{\frac{4}{3}}-1\right)\left(2 \mu^{2}+2 Z^{\frac{4}{3}}-3\right)-\sqrt{6} \lambda X Z^{3}\left(Z^{4}-1\right)^{2}+3 Z^{4}\left(Z^{\frac{4}{3}}-1\right)^{2}\right), \\
& \frac{\mathrm{d} Z}{\mathrm{~d} N}=\frac{3 X^{2}\left(\sqrt{6} \lambda X Z^{4}-6 Z^{7 / 3}+6 Z-\sqrt{6} \lambda\left(2 \mu^{2}+1\right) X\right)}{2\left(X^{2}\left(-2 \mu^{2}+3 Z^{4}-1\right)-Z^{10 / 3}+Z^{2}\right)}
\end{aligned}
$$

where

$$
\begin{gathered}
U_{1}=2 Z^{2}\left(Z^{\frac{4}{3}}-1\right)\left(X^{2}\left(2 \mu^{2}-3 Z^{\frac{4}{3}}+1\right)+Z^{2}\left(Z^{\frac{4}{3}}-1\right)\right) \\
U_{2}=Z^{2} \sqrt{2-2 Z^{\frac{4}{3}}}\left(Z^{\frac{4}{3}}-1\right)\left(X^{2}\left(2 \mu^{2}-3 Z^{\frac{4}{3}}+1\right)+Z^{2}\left(Z^{\frac{4}{3}}-1\right)\right)
\end{gathered}
$$

Note that only two of these are independent since differentiating the constraint one has

$$
2 \frac{X^{\prime}}{X}=2 \frac{Y^{\prime}}{Y}+\frac{4 Z^{\frac{1}{3}}}{3\left(1-Z^{\frac{4}{3}}\right)} \frac{Z^{\prime}}{Z}
$$

It is straightforward to verify that the dynamical system above indeed satisfies this relation.

[1] E. J. Copeland, M. Sami, and S. Tsujikawa, Int. J. Geom. Methods Mod. Phys. D15, 1753 (2006).

[2] B. Ratra and P. J.E. Peebles, Phys. Rev. D 37, 3406 (1988).

[3] I. Zlatev, L.-M. Wang, and P. J. Steinhardt, Phys. Rev. Lett. 82, 896 (1999).

[4] C. Armendariz-Picon, V. F. Mukhanov, and P. J. Steinhardt, Phys. Rev. D 63, 103510 (2001).
[5] S. Weinberg, Phys. Rev. 138, B988 (1965).

[6] T. Clifton, P. G. Ferreira, A. Padilla, and C. Skordis, Phys. Rep. 513, 1 (2012).

[7] J. D. Bekenstein, in Marcel Grossmann Meeting on General Relativity, edited by F. Satō and T. Nakamura (World Scientific Publishing, Singapore, 1992), p. 905.

[8] J. D. Bekenstein, Phys. Rev. D 48, 3641 (1993).

[9] T. S. Koivisto, arXiv:0811.1957. 
[10] M. Zumalacarregui, T. Koivisto, D. Mota, and P. RuizLapuente, J. Cosmol. Astropart. Phys. 05 (2010) 038.

[11] C. Deffayet, S. Deser, and G. Esposito-Farese, Phys. Rev. D 80, 064015 (2009).

[12] C. Deffayet, G. Esposito-Farese, and A. Vikman, Phys. Rev. D 79, 084003 (2009).

[13] C. Deffayet, X. Gao, D. A. Steer, and G. Zahariade, Phys. Rev. D 84, 064039 (2011).

[14] D. Bettoni and S. Liberati, Phys. Rev. D 88, 084020 (2013).

[15] M. Zumalacrregui and J. Garca-Bellido, Phys. Rev. D 89, 064046 (2014).

[16] J. Gleyzes, D. Langlois, F. Piazza, and F. Vernizzi, J. Cosmol. Astropart. Phys. 02 (2015) 018.

[17] X. Gao, Phys. Rev. D 90, 104033 (2014).

[18] X. Gao, Phys. Rev. D 90, 081501 (2014).

[19] C. Deffayet, G. Esposito-Farese, and D. A. Steer, Phys. Rev. D 92, 084013 (2015).

[20] T. Koivisto, D. Wills, and I. Zavala, J. Cosmol. Astropart. Phys. 06 (2014) 036.

[21] C. de Rham and A. J. Tolley, J. Cosmol. Astropart. Phys. 05 (2010) 015.

[22] G. Goon, K. Hinterbichler, A. Joyce, and M. Trodden, Phys. Lett. B 714, 115 (2012).

[23] C. de Rham, Living Rev. Relativity 17, 7 (2014).

[24] G. W. Horndeski, Int. J. Theor. Phys. 10, 363 (1974).

[25] N. Kaloper, Phys. Lett. B 583, 1 (2004).

[26] J. Noller, J. Cosmol. Astropart. Phys. 07 (2012) 013.

[27] M. Zumalacarregui, T. S. Koivisto, and D. F. Mota, Phys. Rev. D 87, 083010 (2013).

[28] C. van de Bruck and G. Sculthorpe, Phys. Rev. D 87, 044004 (2013).

[29] C. van de Bruck, J. Morrice, and S. Vu, Phys. Rev. Lett. 111, 161302 (2013).

[30] P. Brax, C. Burrage, A.-C. Davis, and G. Gubitosi, J. Cosmol. Astropart. Phys. 11 (2013) 001.

[31] P. Brax and C. Burrage, Phys. Rev. D 90, 104009 (2014).

[32] P. Brax and C. Burrage, Phys. Rev. D 91, 043515 (2015).

[33] J. Sakstein, J. Cosmol. Astropart. Phys. 12 (2014) 012.

[34] J. Sakstein, Phys. Rev. D 91, 024036 (2015).

[35] T. S. Koivisto and F. R. Urban, J. Cosmol. Astropart. Phys. 03 (2015) 003.

[36] R. Hagala, C. Llinares, and D. F. Mota, arXiv:1504.07142.

[37] C. van de Bruck and J. Morrice, J. Cosmol. Astropart. Phys. 04 (2015) 036.
[38] G. Domnech, A. Naruko, and M. Sasaki, J. Cosmol. Astropart. Phys. 10 (2015) 067.

[39] P. Brax, P. Brun, and D. Wouters, Phys. Rev. D 92, 083501 (2015).

[40] P. Brax, C. Burrage, and C. Englert, Phys. Rev. D 92, 044036 (2015).

[41] S. Tsujikawa, Phys. Rev. D 92, 064047 (2015).

[42] H. Y. Ip, J. Sakstein, and F. Schmidt, J. Cosmol. Astropart. Phys. 10 (2015) 051.

[43] C. Wetterich, arXiv:1402.5031.

[44] J. D. Bekenstein, Phys. Rev. D 70, 083509 (2004).

[45] A. Coley, Dynamical Systems and Cosmology, Astrophysics and Space Science Library (Springer Netherlands, 2003).

[46] A. Alho and C. Uggla, Phys. Rev. D 92, 103502 (2015).

[47] E. J. Copeland, A. R. Liddle, and D. Wands, Phys. Rev. D 57, 4686 (1998).

[48] E. J. Copeland, A. R. Liddle, and D. Wands, Phys. Rev. D 57, 4686 (1998).

[49] L. Amendola, Phys. Rev. D 62, 043511 (2000).

[50] D. J. Holden and D. Wands, Phys. Rev. D 61, 043506 (2000).

[51] S. Das, P. S. Corasaniti, and J. Khoury, Phys. Rev. D 73, 083509 (2006).

[52] P. Brax and A.-C. Davis, Phys. Lett. B 707, 1 (2012).

[53] P. Brax, A.-C. Davis, and H. A. Winther, Phys. Rev. D 85, 083512 (2012).

[54] P. Brax, A.-C. Davis, and J. Sakstein, Phys. Lett. B 719, 210 (2013).

[55] P. Brax, A.-C. Davis, and J. Sakstein, J. Cosmol. Astropart. Phys. 10 (2013) 007.

[56] A. G. Sanchez et al., Mon. Not. R. Astron. Soc. 425, 415 (2012).

[57] P. Ade et al. (Planck Collaboration), Astron. Astrophys. 571, A1 (2014).

[58] P. Ade et al. (Planck Collaboration), Astron. Astrophys. 571, A16 (2014).

[59] M. Betoule et al. (SDSS), Astron. Astrophys. 568, A22 (2014).

[60] T. E. Collett and M. W. Auger, Mon. Not. R. Astron. Soc. 443, 969 (2014).

[61] R. R. Caldwell, M. Kamionkowski, and N. N. Weinberg, Phys. Rev. Lett. 91, 071301 (2003).

[62] B. Bertotti, L. Iess, and P. Tortora, Nature (London) 425, 374 (2003).

[63] G. Esposito-Farese, AIP Conf. Proc. 736, 35 (2004).

[64] S. Renaux-Petel, S. Mizuno, and K. Koyama, J. Cosmol. Astropart. Phys. 11 (2011) 042. 